\title{
Effect of cabazitaxel on macrophages improves CD47-targeted immunotherapy for triple-negative breast cancer
}

Xu Cao, ${ }^{1}$ Bolei Li, ${ }^{1}$ Jing Chen, ${ }^{1}$ Jessica Dang, ${ }^{1}$ Siqi Chen, ${ }^{1}$ E Gulsen Gunes (D) , ${ }^{1,2}$ Bo Xu, ${ }^{1,2}$ Lei Tian, ${ }^{1,2}$ Sabina Muend, ${ }^{1}$ Mustafa Raoof, ${ }^{3}$ Christiane Querfeld, ${ }^{1,2,4,5}$ Jianhua Yu, ${ }^{1,2,6}$ Steven T. Rosen, ${ }^{2,7}$ Yingyu Wang, ${ }^{8}$ Mingye Feng (D) ${ }^{1}$

To cite: Cao X, Li B, Chen J, et al. Effect of cabazitaxel on macrophages improves CD47targeted immunotherapy for triple-negative breast cancer. Journal for ImmunoTherapy of Cancer 2021;9:e002022. doi:10.1136/jitc-2020-002022

- Additional material is published online only. To view, please visit the journal online (http://dx.doi.org/10.1136/jitc2020-002022).

$\mathrm{XC}, \mathrm{BL}$ and $\mathrm{JC}$ contributed equally.

Accepted 04 February 2021

Check for updates

(C) Author(s) (or their employer(s)) 2021. Re-use permitted under CC BY-NC. No commercial re-use. See rights and permissions. Published by BMJ.

For numbered affiliations see end of article.

Correspondence to

Dr Mingye Feng;

mfeng@coh.org

\section{ABSTRACT}

Background Limited therapeutic options are available for triple-negative breast cancer (TNBC), emphasizing an urgent need for more effective treatment approaches. The development of strategies by targeting tumorassociated macrophages (TAMs) to stimulate their ability of Programmed Cell Removal (PrCR) provides a promising new immunotherapy for TNBC treatment.

Methods CD47 is a critical self-protective "don't eat me" signal on multiple human cancers against macrophage immunosurveillance. Using human and mouse TNBC preclinical models, we evaluated the efficacy of PrCRbased immunotherapy by blocking CD47. We performed high-throughput screens on FDA-approved anti-cancer small molecule compounds for agents potentiating PrCR and enhancing the efficacy of CD47-targeted therapy for TNBC treatment.

Results We showed that CD47 was widely expressed on TNBC cells and TAMs represented the most abundant immune cell population in TNBC tumors. Blockade of CD47 enabled PrCR of TNBC cells, but the efficacy was not satisfactory. Our high-throughput screens identified cabazitaxel in enhancing PrCR-based immunotherapy. A combination of CD47 blockade and cabazitaxel treatment yielded a highly effective treatment strategy, promoting PrCR of TNBC cells and inhibiting tumor development and metastasis in preclinical models. We demonstrated that cabazitaxel potentiated PrCR by activating macrophages, independent of its cytotoxicity toward cancer cells. When treated with cabazitaxel, the molecular and phenotypic signatures of macrophages were polarized toward M1 state, and the NF-kB signaling pathway became activated. Conclusion The combination of CD47 blockade and macrophage activation by cabazitaxel synergizes to vastly enhance the elimination of TNBC cells. Our results show that targeting macrophages is a promising and effective strategy for TNBC treatment.

\section{INTRODUCTION}

Triple-negative breast cancer (TNBC) accounts for $15 \%-20 \%$ of all breast cancers and is a highly aggressive and the most difficult-to-treat subgroup of breast cancers, ${ }^{12}$ commonly associated with a poor patient prognosis and a median survival of approximately 18 months or less. ${ }^{3}$ TNBC is characterized by a lack of estrogen receptor (ER), progesterone receptor (PR) and human epidermal growth factor receptor 2 (HER2) expression, all of which are important therapeutic targets. ${ }^{1} 2$ TNBC is considered a single disease entity, with chemotherapy remaining the mainstay and standard treatment approach. However, further long-term clinical benefits have thus far been hindered by a poor prognosis and higher relapse rate caused by lingering cancer cells that were either not eradicated by chemotherapy, or had developed a resistance to it.

The understanding of the mechanisms by which the immune cells detect and attack cancer cells has enabled the development of effective immunotherapies against a range of cancers. ${ }^{4-10}$ A fraction of TNBC tumors upregulate programmed death ligand 1 (PDL1) and are infiltrated with lymphocytes, providing the rationale for using immunotherapy for TNBC treatment. ${ }^{11}$ Clinical trials in advanced TNBC using atezolizumab, an antibody targeting PD-L1 that effectively blocks the interaction between PD-L1 and the immune checkpoint $\mathrm{PD}-1$, resulted in significantly improved overall survival and progression-free survival. ${ }^{3} 13$ As a result, combinatory therapy using both atezolizumab and nab-paclitaxel has been recently approved by the Food and Drug Administration (FDA) in 2019 for the treatment of advanced TNBC. ${ }^{1415}$ Atezolizumab currently remains the only FDA-approved immunotherapeutic agent for the treatment of TNBC. While substantial clinical benefits have been observed in patients receiving atezolizumabnab-paclitaxel combination therapy, only a small fraction of patients showed a positive response, ${ }^{3}$ likely due to the high level of heterogeneity of TNBC tumors. ${ }^{13} 16 \quad 17$ Coupled with an overall poor patient prognosis, a limited number of therapeutic options 
for this aggressive disease has instilled urgency for developing novel efficacious therapies.

During cancer development, circulating monocytes are constantly recruited to primary tumors and metastatic sites where they derive to macrophages. ${ }^{18} 19$ Tumor-associated macrophages (TAMs) represent the most abundant cell type in the tumor microenvironment, composing up to $50 \%$ of tumor mass in almost every type of solid cancer, including breast cancer. ${ }^{18-21}$ Targeting TAMs by activating them to attack malignant cells or inhibiting their function in supporting tumor progression, represents a new class of cancer treatment approaches. While directly depleting TAMs inhibited tumor growth in certain cancers, reprogramming TAMs to elicit their potent tumoricidal functions has become a highly attractive strategy bearing significant therapeutic potential, thus ushering in the next generation of cancer immunotherapy. ${ }^{22}$

Recent exciting breakthroughs in cancer immunology have begun to unveil the role of macrophages in directly recognizing and engulfing cancer cells, a process termed "Programmed Cell Removal" (PrCR). ${ }^{23-25}$ Cancer cells and macrophages communicate with each other, during which an imbalance of pro-phagocytic "eat me" pathways over anti-phagocytic "don't eat me" pathways results in a downstream signaling cascade in macrophages. ${ }^{23-25}$ Subsequently, cytoskeleton rearrangement drives the engulfment of target cells by macrophages, and engulfed cells are digested in the lysosomes. PrCR is an efficient process of cancer immunosurveillance which can be initiated independent of the induction of cancer cell death. ${ }^{23-25}$ In tumors, malignant cells have developed self-protective mechanisms to facilitate their survival, such as the upregulation of "don't eat me" signals, which transduce negative signaling to macrophages, therefore preventing PrCR. The blockade of such signals reinstates macrophage-mediated PrCR, leading to an effective elimination of cancer cells. To date, several potential "don't eat me" signals have been identified, including CD24, CD47 and MHC I. ${ }^{26-29}$ Among these, CD47 has been demonstrated to be the most important "don't eat me" signal. CD47 is ubiquitously expressed and particularly upregulated on a variety of human cancer cells, functioning via a cis-interaction with Signal Receptor Protein alpha (SIRP $\alpha$ ) on macrophages to inhibit PrCR by effectively shutting down the machinery required to carry out PrCR. ${ }^{262730}$ A blockade of CD47 has been shown to be effective in inducing PrCR, therefore eliciting potent anti-cancer effects in multiple preclinical cancer models with promising benefits in clinical trials when used in both monotherapy and combinatory therapy. ${ }^{26} 2730-37$

Despite the encouraging progress, it remains to be explored whether the induction of PrCR can be used as a treatment approach for TNBC. CD47 was reported to be highly expressed on TNBC tumors and its expression was correlated with worse patient prognosis and outcomes. ${ }^{38}$ In this study, using TNBC preclinical models, we examined the efficacy and potential of treating TNBC with therapies aiming to induce PrCR. We demonstrated that although a blockade of CD47 was sufficient in inducing PrCR of TNBC cells, its efficacy was not satisfactory. We further identified that when used in combination with CD47 blockade, cabazitaxel, an FDA-approved chemotherapeutic agent, ${ }^{39}$ elicited strong anti-cancer effects on PD-L1+ and PD-L1- TNBC. This treatment regimen was thus termed "enhanced PrCR therapy". More importantly, we discovered that cabazitaxel functions by directly promoting the ability of macrophages to perform PrCR independently of its cytotoxicity toward cancer cells. Furthermore, we identified that NF-kB signaling plays a key role in transcriptional landscape remodeling of these macrophages. Our discoveries uncovered a novel function and purpose for cabazitaxel in PrCR-based immunotherapy and highlighted the potential for PrCR induction to be a promising new direction for developing novel and more effective therapies to fight TNBC.

\section{MATERIALS AND METHODS \\ Mice}

$\mathrm{BALB} / \mathrm{c}$ and $\mathrm{RAG}^{-/-} \gamma_{\mathrm{c}^{-/-}} \mathrm{BALB} / \mathrm{c}$ mice were bred in the Animal Resources Center at City of Hope Comprehensive Cancer Center. BALB/c mouse strain was purchased from the Jackson Laboratory. RAG2 ${ }^{-/-} \mathrm{\gamma c}^{-/-}$mouse strain was a generous gift from Dr. Irving L. Weissman at Stanford University. All animal procedures were in accordance with the guidelines and approved by the Administrative Panel on Laboratory Animal Care at City of Hope Comprehensive Cancer Center.

\section{Cell culture}

Human triple-negative breast cancer cell lines (MDA-MB231, MDA-MB-453, MDA-MB-468), a mouse triple-negative breast cancer cell line (4T1), human non-Hodgkin's lymphoma cell lines (Raji and Mac1) and a mouse monocyte/macrophage cell line (RAW264.7) were used in this study. MDA-MB-231, MDA-MB-453, MDA-MB-468, 4T1, Raji and RAW264.7 were purchased from ATCC. Mac1 was a generous gift from Dr. Marshall Kadin at Roger Williams Medical Center. Cryopreservation of large quantities of low passage (below 3) cells was performed and cells with passage number below 20 were used in this study. Cells were routinely cultured in high-glucose DMEM medium supplemented with $10 \%$ fetal bovine serum (MDA-MB231, MDA-MB-453, MDA-MB-468 and RAW264.7) or RPMI-1640 medium supplemented with 10\% fetal bovine serum (4T1, Raji and Mac1). All cell lines were maintained at $37^{\circ} \mathrm{C}$ in a humidified $5 \% \mathrm{CO}_{2} / 95 \%$ air incubator. Mycoplasma examination was routinely performed every 2 months.

\section{Analysis of clinical datasets}

Gene expression datasets collected by The Cancer Genome Atlas (TCGA) were obtained from UCSC Xena (https://xenabrowser.net $/)^{40}$ in the form of $\log _{2}$ (normalized counts +1 ) values with the query "TCGA Breast Cancer (BRCA)". From the dataset, the TNBC 
patient group was selected by the deficiency of ER, PR and HER2. Expression of CD47 and PD-L1 was analyzed and compared between non-TNBC and TNBC groups.

For analysis of immune composition in TNBC tumors, four independent studies were included into the analysis. Gene expression datasets of these studies (GSE25066; GSE58812; GSE76124; GSE21653 $)^{41-44}$ were obtained from Gene Expression Omnibus (GEO) (https://www. ncbi.nlm.nih.gov/geo/). The analytical tool CIBERSORT (https://cibersort.stanford.edu/) ${ }^{45}$ was used to estimate the relative proportion of the immune cell types.

To analyze the correlation of overall survival and the ratio of M1-like to M2-like TAMs in TNBC patients, TNBC samples from the TCGA dataset were deconvoluted by CIBERSORT. ${ }^{45}$ The samples were grouped by ratio of M1-like to M2-like TAMs and survival analysis was performed with overall survival information of each patient.

\section{CRISPR/Cas9-mediated gene editing}

The CRISPR/Cas9 system was used for suppressing gene expression in TNBC cells. Pairs of primers containing sequences of control sgRNAs or sgRNAs targeting human CD47, mouse CD47, mouse TLR2 or mouse TLR4 genes were designed and cloned into the all-in-one LentiCRISPR V2 vector. ${ }^{46}$ The LentiCRISPR V2 vector was transfected with the packing plasmids into $293 \mathrm{~T}$ cells to generate lentiviruses. Viruses were collected 48-72 hours after transfection and filtered through $0.45 \mu \mathrm{m}$ filters to remove residual cells and cell debris. For the infection, target cells were incubated with lentiviruses for 48 hours in the presence of polybrene $(8 \mu \mathrm{g} / \mathrm{mL})$ and selected with puromycin $(2 \mu \mathrm{g} / \mathrm{mL}) 24$ hours after removal of the viruses.

The following sgRNA sequences were used:

Control1 (Non-target):GAACGUAGAAAUUCCCAUUU ${ }^{46}$ Control 2 (LacZ): UUGGGAAGGGGGAUCGGUGC ${ }^{47}$ Human CD47: CUACUGAAGUAUACGUAAAG ${ }^{46}$ Mouse CD47: CCCUUGCAUCGUCCGUAAUG ${ }^{46}$ Mouse TLR2: CGCGGAUCGACUUUAGACUU ${ }^{46}$ Mouse TLR4: ACACGUCCAUCGGUUGAUCU ${ }^{46}$

\section{Flow cytometry analysis}

Anti-mouse CD47 (clone miap301; BioLegend), antihuman $\mathrm{CD} 47$ (clone B6H12; BD Biosciences), antimouse F4/80 (clone BM8; BioLegend), anti-mouse/ human CD11b (clone M1/70; BioLegend), anti-mouse MHC-II (clone M5/114.15.2; BioLegend), anti-mouse CD206 (clone C068C2; BioLegend), anti-mouse Sirp $\alpha$ (clone P84; BioLegend) and anti-human CD206 (clone 15-2; BioLegend) were used for FACS analyses. Antibodies were phycoerythrin (PE), PE Cy7, APC, APC Cy7, Alexa Fluor 700, BV787, or BV605 conjugated, or fluorophore-conjugated secondary antibodies were used. Sytox blue was used to exclude dead cells. Annexin V (BD Biosciences) and 7-aminoactinomycin D (7-AAD; ThermoFisher) were used to evaluate cell viability of macrophages on cabazitaxel treatment. Flow cytometry was performed using the BD LSRFortessa cell analyzers.

\section{Generation of macrophages}

To generate mouse bone marrow-derived macrophages, $\mathrm{BALB} / \mathrm{c}$ mice 6 to 12 weeks old were euthanized and femurs were flushed with a 25G needle and filtered through a $70 \mu \mathrm{m}$ strainer. The filtrates were pelleted by centrifugation and red blood cells were lysed with ACK lysis buffer at room temperature for $2 \mathrm{~min}$ and washed with IMDM supplemented with 10\% FBS. Subsequently, cells were suspended in IMDM supplemented with $10 \%$ FBS and $10 \mathrm{ng} / \mu \mathrm{L}$ murine MCSF. Macrophages from day 6 to day 8 were used for functional assays.

To generate human peripheral blood-derived macrophages, monocytes were enriched from human peripheral blood by magnetic-activated cell sorting using Whole Blood CD14 microbeads (Miltenyi). CD14 $4^{+}$monocytes were cultured in IMDM supplemented with $10 \%$ human serum. Macrophages from day 6 to day 8 were used for functional assays.

\section{Phagocytosis assay}

Macrophage-based phagocytosis was examined by flow cytometry-, luminescence- or microscopy-based assays.

Macrophages were harvested by TrypLE and scrapers, and equally divided into FACS tubes with $1 \times 10^{5}$ cells per tube. Target cancer cells were either GFP-expressing or labeled with cell-permeant calcein AM. For calcein labeling, cells were incubated with $200 \mathrm{nM}$ calcein $\mathrm{AM}$ in PBS at $37^{\circ} \mathrm{C}$ for $20 \mathrm{~min}$ and washed twice with DMEM medium supplemented with 10\% FBS. Cancer cells were added and mixed with macrophages with the addition of antibodies (anti-CD47, BD Biosciences; anti-Sirp $\alpha$, BioLegend) or IgG control (BioLegend), and incubated at $37^{\circ} \mathrm{C}$ for $1-2$ hours. Anti-CD47 clone B6H12 was used for most experiments and clone CC2C6 was used for the experiment in online supplemental figure 3E. Macrophages were stained with anti-F4/80 antibody conjugated with PE-Cy7. The phagocytosis index was quantified as the percentage of macrophages that phagocytosed cancer cells during the incubation, namely the ratio of $\mathrm{GFP}^{+} \mathrm{PE}-\mathrm{Cy} 7^{+}$cells to $\mathrm{PE}-\mathrm{Cy} 7^{+}$cells. For pre-treatment experiments, cancer cells or macrophages were pretreated with various concentrations of cabazitaxel for 24 hours followed by phagocytosis assays. Phagocytosis index was normalized to the maximal response in the experiments. In indicated experiments, phagocytosis of cancer cells was also examined with fluorescence microscopy.

For examining the long-term effects of phagocytosis, the phagocytic clearance of cancer cells mediated by macrophages was quantified by detection of the luminescence signals from surviving cancer cells after coculture with macrophages. Specifically, luciferase equipped cancer cells were co-cultured with macrophages for 24 hours in the presence or absence of antibodies and/or cabazitaxel. Wells containing cancer cells alone (without macrophage addition) were used as a control for calculation 
(phagocytosis rate $=0 \%$ ). Luciferin was added and the luminescence signals were read with Cytation 3 which were used to quantify the remaining live cancer cells. The phagocytosis rate was calculated as the ratio of signals in the treatment group to the signal of wells containing cancer cells alone. Phagocytosis index was normalized to the maximal response in the experiments.

\section{Mouse models}

MDA-MB-231 cells expressing a luciferase-eGFP fusion protein were used for the human TNBC tumor model in $\mathrm{RAG}^{-/-} \mathrm{\gamma c}^{-/-}$mice. Cells were suspended in high glucose DMEM medium containing 25\% Corning Matrigel Matrix (v/v) and injected to the mammary fat pad of $\mathrm{RAG2}^{-/-} \mathrm{\gamma c}^{-/-}$mice with $5 \times 10^{5}$ cells per mouse. Nine days after the engraftment, the mice were treated with vehicle (Control), CD47-blocking antibody (clone B6H12; BioXCell), cabazitaxel (Cayman Chemical), or both, once every week. CD47-blocking antibodies were administered intravenously ( $4 \mathrm{mg} / \mathrm{kg}$ body weight), and cabazitaxel was administered intratumorally ( $40 \mu \mathrm{g} / \mathrm{kg}$ body weight).

Raji cells expressing a luciferase-eGFP fusion protein were used for the human NHL tumor model in RAG2 ${ }^{-/-}$ $\gamma_{\mathrm{c}^{-/-}}$mice. Cells were suspended in high glucose DMEM medium containing 25\% Corning Matrigel Matrix (v/v) and injected subcutaneously on the back of RAG2 ${ }^{-/-}$ $\gamma^{-/-}$mice with $5 \times 10^{5}$ cells per mouse. Nine days after the engraftment, the mice were treated with vehicle (Control) or CD47-blocking antibody (clone B6H12; BioXCell) once every week. CD47-blocking antibodies were administered intravenously ( $4 \mathrm{mg} / \mathrm{kg}$ body weight).

$\mathrm{Ctrl}^{\mathrm{KD}}$ and $\mathrm{CD} 47^{\mathrm{KD}} 4 \mathrm{~T} 1$ cells were used for the mouse TNBC tumor model in immune competent mice. Cells were suspended in RPMI-1640 medium containing 25\% Corning Matrigel Matrix (v/v) and injected to the mammary fat pad of BALB/c mice with $5 \times 10^{4}$ cells per mouse. Nine days after the engraftment, the mice were treated with vehicle or cabazitaxel $(80 \mu \mathrm{g} / \mathrm{kg}$ body weight) intratumorally, twice every week.

$\mathrm{Ctrl}^{\mathrm{KD}}$ and $\mathrm{CD} 47^{\mathrm{KD}} 4 \mathrm{~T} 1$ cells expressing a luciferaseeGFP fusion protein were used for the mouse metastatic TNBC model. Cells were suspended in RPMI-1640 medium and injected intravenously to BALB/c mice with $5 \times 10^{5}$ cells per mouse. Three days after the engraftment, when lung colonization by $4 \mathrm{~T} 1$ cells was confirmed by bioluminescence imaging, the mice were treated with vehicle or cabazitaxel ( $2 \mathrm{mg} / \mathrm{kg}$ body weight) intravenously, twice every week for up to 3 weeks, as indicated in Results section. For cabazitaxel treatment, the formulations of vehicle are $5 \%$ ethanol $+5 \%$ polysorbate- $80+90 \%$ of glucose in sterile water $(\mathrm{v} / \mathrm{v})$.

Tumor cell engraftment and growth in vivo were monitored by bioluminescent imaging. D-Luciferin potassium salt (Biosynth Carbosynth) was dissolved in PBS to a final concentration of $16.6 \mathrm{mg} / \mathrm{mL}$. Mice were injected intraperitoneally with luciferin solution at a dose of 0.139 g luciferin $/ \mathrm{kg}$ body weight. Bioluminescent imaging was performed at indicated time points. Images were captured and analyzed with a Lago X (Spectral Instruments Imaging) and bioluminescence signals were analyzed with Aura Image software.

\section{Tumor-associated macrophage analysis}

$\mathrm{BALB} / \mathrm{c}$ mice were engrafted with $4 \mathrm{~T} 1$ cells by injection to the mammary fat pad and treated with vehicle or cabazitaxel. The resultant tumors were collected, minced into pieces with diameters less than $1 \mathrm{~mm}$, and dissociated in DMEM with Liberase TM enzymes (ThermoFisher) and DNase I (ThermoFisher) at $37^{\circ} \mathrm{C}$ for 1 hour to achieve a single-cell suspension. Cells were pelleted by centrifugation and red blood cells were lysed with ACK lysis buffer, washed twice with FACS buffer (PBS with 2\% FBS) and filtered through a $70 \mu \mathrm{m}$ cell strainer. Samples were treated with FcR blocker (Miltenyi) before being stained with the indicated antibodies, and subjected to flow cytometry analysis. Tumor-associated macrophages were gated as $\mathrm{CD}_{4} 5^{+}, \mathrm{CD} 11 \mathrm{~b}^{+}, \mathrm{F} 4 / 80^{+}$and Zombie Violet ${ }^{-}$. The expression of MHC-II and CD206 on TAMs was examined.

\section{Macrophage depletion}

$\mathrm{BALB} / \mathrm{c}$ mice were injected with $200 \mu \mathrm{L}$ of control (PBS) liposomes or clodronate liposomes (Liposoma) intravenously 1 day before the transplantation of 4T1 cells, and $100 \mu \mathrm{L}$ of the control liposomes or clodronate liposomes were injected every 4 days thereafter throughout the duration of the experiment. The mice were engrafted with $\mathrm{Ctrl}{ }^{\mathrm{KD}}$ or $\mathrm{CD} 47^{\mathrm{KD}} 4 \mathrm{~T} 1$ cells by injection to the mammary fat pad and treated with vehicle or cabazitaxel. To verify the efficacy of macrophage depletion, the resultant tumors were collected, dissociated to single-cell suspension, and subjected to flow cytometry analysis. Tumorassociated macrophages were gated as $\mathrm{CD} 11 \mathrm{~b}^{+}, \mathrm{F} 4 / 80^{+}$.

\section{Bone marrow-derived macrophage (BMDM) polarization}

BMDMs or RAW264.7 cells were seeded in 12-well plates at a density of $1.5 \times 10^{6}$ cells per well and stimulated for 48 hours with medium for M0-like, LPS $(50 \mathrm{ng} / \mathrm{mL})$ and IFN $\gamma(10 \mathrm{ng} / \mathrm{mL})$ for M1-like, or IL4 $(10 \mathrm{ng} / \mathrm{mL})$ for M2-like polarization.

\section{NF-kB reporter assay}

The GFP-based NF-kB reporter was a generous gift from Dr. Susan Carpenter at the University of California, Santa Cruz. In this system, 5x NF-kB-binding motifs was upstream of the minimal CMV promoter for driving GFP expression. ${ }^{48}$ We generated a luminescence-based NF-kB reporter by cloning the firefly luciferase gene into this vector to replace the GFP gene. RAW 264.7 cells were infected with lentiviruses expressing the luminescencebased NF-kB reporter to generate the reporter line. $\mathrm{Ctrl}^{\mathrm{KD}}{ }^{\mathrm{K}} \mathrm{TLR}^{\mathrm{KD}}$ or $\mathrm{TLR} 4^{\mathrm{KD}}$ RAW264.7 NF-kB reporter lines were cultured in 96-well plates overnight, and then stimulated with LPS $(10 \mathrm{ng} / \mathrm{mL})$ or cabazitaxel $(20,10$, $5,2.5 \mu \mathrm{M})$ for another 8 hours. Unstimulated cells were used as the negative control. Luciferin was added to the cells and luminescence signals were measured using a luminometer (Cytation3 imaging reader, BioTek). 


\section{RNA sequencing}

BMDMs from BALB/c mice after 7 days of differentiation were treated with DMSO (control) or $10 \mu \mathrm{M}$ cabazitaxel for 24 hours, after which total RNA was extracted with an RNA extraction kit (Qiagen) and used for library preparation. BMDMs were generated from three individual mice for triplicate samples. The samples were submitted to Novogene for RNA sequencing. The libraries were sequenced on the Illumina NovaSeq 6000 platform. Clean reads were generated after removing reads containing adapter or poly-N and reads of low quality. Next, mapped reads were obtained through aligning clean reads to the reference genome mm10 using STAR V.2.5.3a. The number of mapped clean reads was then counted and normalized into fragments per kilobase of transcript per million reads (FPKM). FPKM value of 17,891 genes obtained through the RNA-seq were used as the original raw data and analyzed by GSA algorithm to find differentially expressed genes, presented by the volcano plot. The genes located out of the borders of the lines (fold change $\mathrm{FC}>2$ and $\mathrm{p}$ value $<0.05$ ) were considered to be differentially expressed genes (DEGs). In this data set, 452 DEG genes were upregulated and 491 DEG genes were downregulated after treated by cabazitaxel. Gene Set Enrichment Analysis was performed using the GSEA software and the Molecular Signatures Database. ${ }^{49} 50$

\section{Multidimensional scaling (MDS) analysis}

Microarray raw data GSE69607 and RNA sequencing raw count data GSE103958 were downloaded from NCBI GEO separately as reference 1 and reference 2 gene expression of M0, M1 and M2 macrophages. ${ }^{51}{ }^{52}$ For reference 1, the expression of 20,556 genes was extracted and annotated by Affymetrix Mouse Genome 430 V.2.0 using R package affy (V.1.62.0), and gcrma (V.2.56.0) for background noise removal and $\log 2$ transformation. The microarray gene expression was scaled to have zero mean and unit variance, and quantile normalized before performing MDS. For reference 2, the expression of 16,617 genes was extracted, $\log 2$ transformed and scaled to have zero mean and unit variance, and normalized to reference 1 target distribution using FSQN (feature-specific quantile normalization, https://github.com/jenniferfranks/ FSQN) to remove cross-platform batch effects before performing MDS. Together we have $6 \mathrm{M} 0,15 \mathrm{M} 1$ and 8 M2 macrophage reference samples.

Our RNA sequencing fastq files were aligned to Mouse Genome mm10 using STAR V.2.5.3a, and then quantified by genes to mm10-Ensembl Transcripts release 100 on Partek Flow platform. The expression of 17,891 genes was $\log 2$ transformed and scaled to have zero mean and unit variance, and normalized to reference 1 target distribution using FSQN.

MDS was performed with cmdscale function in R3.6.0, and the number of retained dimensions was set to 3 to cluster the M0/M1/M2 macrophage samples, then visualized in 3D dimension using R package ggfortify (V.0.4.10).

\section{High-throughput screen}

The FDA-approved oncology drugs set including 147 current approved anti-cancer small molecules was obtained from the Division of Cancer Treatment \& Diagnosis at the National Cancer Institute (NCI). For the highthroughput screen, individual anti-cancer small molecule compounds were incubated with $0.03 \times 10^{6}$ MDA-MB-231 cells expressing GFP-luciferase and $0.06 \times 10^{6}$ macrophages in the presence or absence of $\mathrm{CD} 47$ blockades (anti-CD47 or anti-Sirp $\alpha$ antibodies) in 96-well plates for 24 hours. Thereafter, luciferin was added into the plate and luminescence signal was measured with Cytation 3. Wells containing $10 \mu \mathrm{M}$ drug and 231 cells were used as a control for calculating phagocytosis rate (phagocytosis rate $=0 \%$ ). The normalized phagocytosis index was calculated as the ratio of phagocytosis rate induced by drug treatment to that by DMSO.

\section{Cytokine/chemokine analysis}

BMDM cells were seeded in 24-well plates at a density of $5 \times 10^{5}$ cells per well and incubated with DMSO (control) or cabazitaxel $(10 \mu \mathrm{M})$. Cell culture medium without BMDM cells (negative control), DMSO-treated BMDM conditioned medium and cabazitaxel-treated BMDM conditioned medium were collected and centrifuged. The supernatants were collected and submitted to Eve Technologies for Mouse Cytokine Array/Chemokine Array 44-plex discovery arrays which were analyzed using Millipore MILLIPLEX mouse cytokine/chemokine kit.

\section{Statistical analysis and graphing software}

All data were statistically analyzed and graphed using Microsoft Excel or GraphPad Prism V.8. Unpaired t-test or ANOVA test was employed to detect the significant differences between groups. Overall survival was evaluated with the Kaplan-Meier method and log-rank test was used for comparisons between groups. The quantitative data in the study were expressed as the mean $\pm \mathrm{SD}$. P values below 0.05 were considered statistically significant differences.

\section{RESULTS \\ CD47 is a therapeutic target in TNBC}

We first evaluated whether CD47 can be harnessed as a valid therapeutic target for inducing PrCR of TNBC. Successful PrCR-based therapies, involving a blockade of $\mathrm{CD} 47$, rely on abundant macrophage infiltration in TNBC tumors as well as CD47 expression on TNBC cells. First, we analyzed RNA sequencing data of four cohorts of TNBC patient specimens using the CIBERSORT analysis, which allowed us to quantify different immune cell populations in the tumor microenvironment. We found that all of the TNBC tumor specimen cohorts examined were highly infiltrated with TAMs, T cells, and plasma cells, with TAMs representing the most abundant immune cell population (figure 1A). Next, we analyzed the gene expression landscape of tumor samples of patients with breast cancer, including both TNBC and 

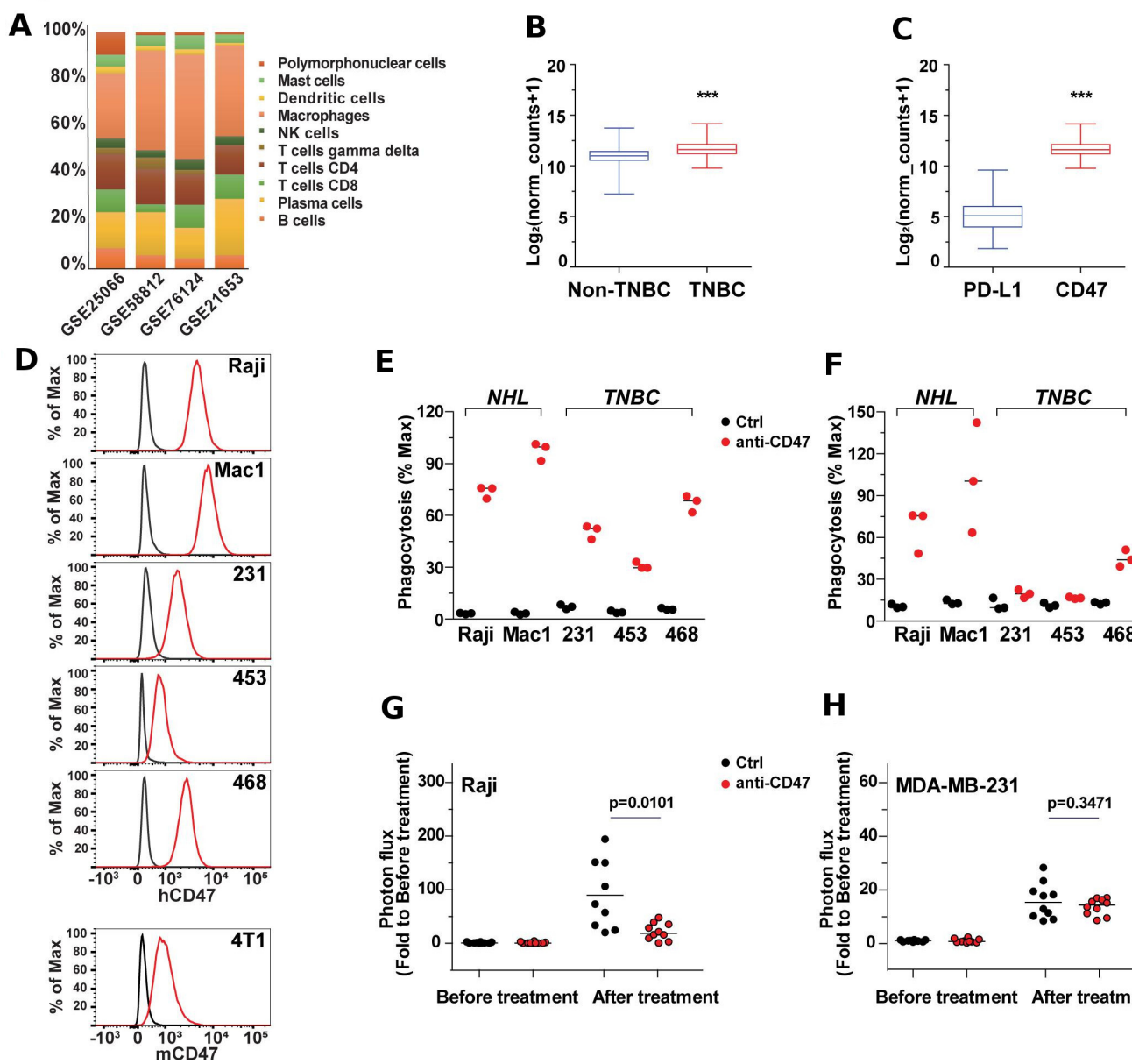

E
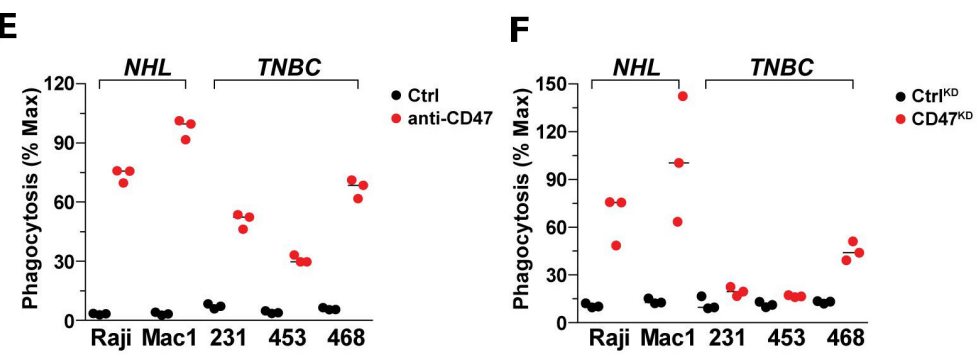

G

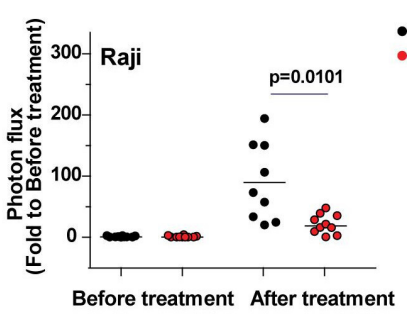

H

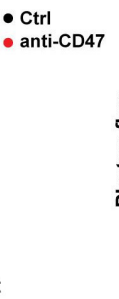

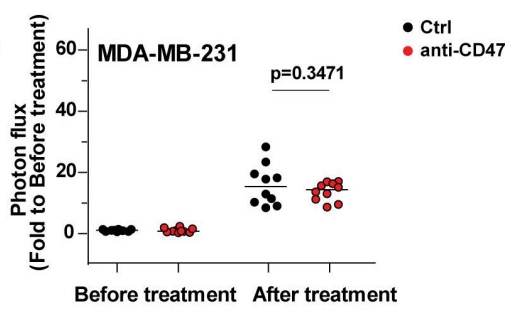

Figure $1 \mathrm{CD} 47$ is a therapeutic target in triple-negative breast cancer (TNBC). (A) Inferred composition of 10 immune cell subsets in TNBC biopsies. The gene signature datasets were obtained from Gene Expression Omnibus (GEO) database. The results were analyzed using CIBERSORT. (B) A comparison of CD47 gene expression between non-TNBC $(n=974)$ and TNBC $(n=123)$, basing on TCGA Breast Cancer (BRCA). Whiskers represent min-max values. ${ }^{* \star *} p<0.001$ (t-test). (C) A comparison of gene expression of PD-L1 and CD47 on TNBC ( $n=123)$, basing on TCGA Breast Cancer (BRCA). Whiskers represent min-max values. ${ }^{* \star *} \mathrm{p}<0.001$ (t-test). (D) Representative histogram plots showing CD47 expression on NHL cell lines (Raji and Mac-1) and TNBC cell lines (MDA-MB-231, MDA-MB-453, MDA-MB-468 and 4T1). Anti-hCD47 (clone B6H12) or anti-mCD47 (clone miap301) were used for human (Raji, Mac-1, MDA-MB-231, MDA-MB-453 and MDA-MB-468) or mouse (4T1) lines. (E) An in vitro flow cytometry-based phagocytosis assay comparing the efficiency of blocking CD47 with an anti-CD47 antibody between TNBC cell lines (MDA-MB-231, MDA-MB-453 and MDA-MB-468) and NHL cell lines (Raji and Mac-1). Mouse M0 bone marrow-derived macrophages (BMDMs) were used for the assay. Phagocytosis was normalized to the maximal response in the experiments. (F) An in vitro flow cytometry-based phagocytosis assay comparing the efficiency of blocking CD47 by knocking down CD47 gene expression between TNBC cell lines (MDA-MB-231, MDA-MB-453 and MDA-MB-468) and NHL cell lines (Raji and Mac-1). Mouse M0 BMDMs were used for the assay. Phagocytosis was normalized to the maximal response in the experiments. ( $G$ and $H$ ) In vivo tumor engraftment assay of Raji and MDA-MB-231 cells. Mice engrafted with Raji or MDA-MB-231 cells were treated with vehicle or CD47-blocking antibody. Tumor engraftment and growth were measured by bioluminescence imaging. $\mathrm{p}=0.0101$ (Raji) or 0.3471 (MDA-MB-231) (t-test).

non-TNBC. We found that CD47 was highly expressed in breast tumors and was more highly expressed in TNBCs than non-TNBCs (figure 1B). When compared with PD-L1 transcripts, a higher number of CD47 transcripts were detected in TNBCs (figure 1C). We then examined several representative human and mouse TNBC preclinical cell models, including MDA-MB-231, MDA-MB-453, MDA-MB-468 and 4T1. We were able to confirm CD47 protein expression on all cell lines, but PD-L1 expression was not detected on MDA-MB-453 and MDA-MB-468 (figure 1D; online supplemental figure 1A). Interestingly, when compared with CD47 expression on hematopoietic cancers such as lymphoma (figure 1D), TNBC expression of $\mathrm{CD} 47$ was relatively lower.

We then performed two layers of experiments to investigate PrCR induced by CD47 blockade: in vitro phagocytosis assays to examine direct PrCR of cancer cells by macrophages and in vivo tumor engraftment assays to examine the anti-cancer effect of $\mathrm{CD} 47$ blockade in tumor development. Bone marrow progenitor cells and monocytes are the main sources for macrophages in the tumors. ${ }^{19}$ Previous studies measuring binding affinity between CD47 and Sirp $\alpha$ demonstrated that Sirp $\alpha$ molecules on macrophages from BALB/c or NSG mice display 
comparable or stronger binding to human CD47, as compared with human Sirp $\alpha$ (Kd for binding of human CD47 to human, BALB/c, NSG or C57BL/ 6 Sirp $\alpha$ are $290,308,2.5,5 \times 10^{10} \mathrm{nM}$, respectively) ${ }^{53-55}$ highlighting BMDMs from these strains as sound research models for assessing the response of cancer cells to PrCR. In this study, we used BMDMs from BALB/c mice for in vitro studies and mice with BALB/c background for evaluation of the efficacy of CD47 blockade in vivo. An additional macrophage model, human peripheral blood monocyte-derived macrophages, was also included for evaluating our findings. On blockade of CD47, whether via antibody or via CRISPR/Cas9-mediated gene editing (online supplemental figure 1B), all the TNBC cell lines examined became prone to macrophage recognition and phagocytosis (figure 1E,F). Consistent with previous studies, a higher phagocytosis rate was observed with CD47-blocking antibody compared with that induced by $\mathrm{CD} 47^{\mathrm{KD}}$, which is due to an additional effect by Fc-region of the antibody. ${ }^{26} 5657$ However, when quantifying PrCR efficiency, we found that TNBC cells were generally less susceptible to macrophage PrCR, as compared with lymphoma cells (figure 1E,F). When we examined the anti-cancer effects of CD47 blockade-induced PrCR in in vivo xenotransplantation models with $\mathrm{RAG2}^{-/-}, \mathrm{\gamma c}^{-/-}$ mice (T-, B- and NK-deficient, with functional phagocytes maintained ${ }^{23}$ ), we found that the response of MDA-MB231 (TNBC) cells to CD47-blocking antibody was not as significant as that of Raji cells (non-Hodgkin's lymphoma) (figure $1 \mathrm{G}, \mathrm{H}$ ).

\section{High-throughput screens with FDA-approved anti-cancer small molecule compounds identify cabazitaxel as a PrCR- promoting agent}

We reasoned that PrCR-based therapy may be effectively applied to TNBC through increasing the susceptibility of TNBC cells to PrCR, or promoting macrophages toward a more phagocytosis-capable phenotype. It follows that therapeutic agents eliciting these particular effects may be combined with a $\mathrm{CD} 47$ blockade to yield a superior efficacy of macrophage-mediated TNBC elimination. The FDA has so far approved more than 100 anti-cancer small molecule compounds as chemotherapy or target therapy agents. In addition to their cytotoxicity, some of these anti-cancer drugs were reported to have immunomodulatory effects, ${ }^{589}$ but whether these small molecule compounds can be combined with PrCR-based therapy for TNBC has not been explored. To this end, we were motivated to investigate such a possibility by screening a library of 147 FDA-approved anti-cancer small molecule compounds obtained from the NCI. We established a high-throughput screening system in which macrophages and MDA-MB-231 cells were co-cultured, and CD47blocking or SIRP $\alpha$-blocking antibodies were used to induce PrCR (figure 2A,B). PrCR efficacy was evaluated by quantifying the number of cancer cells surviving PrCR. MDA-MB-231 cells were equipped with a GFP-luciferase reporter, such that the surviving cells can be precisely quantified by measuring luminescence upon the addition of luciferin. Results from screens with CD47-blocking and SIRPo-blocking antibodies showed a strong correlation with each other, confirming that the enhancement or attenuation of cancer cell survival resulting from the addition of the small molecular compounds were due to the inhibition or promotion of PrCR (figure 2C). Cabazitaxel, a chemotherapeutic agent approved by FDA for prostate cancer treatment, was identified as the most effective agent that dramatically enhanced the efficacy of CD47 blockade-induced PrCR of MDA-MB-231 cells (figure 2B). Cabazitaxel is a taxane which inhibits cell proliferation by promoting microtubule polymerization and thus inhibiting mitosis. Cabazitaxel demonstrated a decreased binding to P-glycoprotein, a frequent cause for resistance to other taxanes, ${ }^{39}$ and excellent intratumoral penetration ability, as shown in preclinical studies and clinical trials. ${ }^{60} 61$ Therefore, we decided to verify our screening results by examining the effects of cabazitaxel in enhancing PrCR in a dose-dependent manner on multiple TNBC lines. Cabazitaxel was able to significantly promote CD47-blocking antibody-induced PrCR at a concentration of $1.25 \mu \mathrm{M}$ or higher for MDA-MB-231, MDA-MB-453 and MDA-MB-468 cells (figure 2D,E). Interestingly, at the concentrations in which PrCR was strongly promoted, cabazitaxel only demonstrated minor cytotoxicity toward MDA-MB-231 cells (figure 2D).

\section{Cabazitaxel promotes PrCR by directly activating macrophages and independently of its cytotoxic effects toward TNBC cells}

Given that cabazitaxel promoted PrCR of TNBC cells without directly inducing cell death, we further hypothesized that cabazitaxel functioned by either inhibiting anti-PrCR mechanisms of TNBC cells or by potentiating the phagocytic capacity of macrophages. To test this hypothesis, we devised two treatment protocols for a phagocytosis assay: (1) cabazitaxel pre-treated BMDMs co-cultured with untreated MDA-MB-231 cells or (2) cabazitaxel pre-treated MDA-MB-231 cells co-cultured with untreated BMDMs. We showed that the pre-treatment of MDA-MB-231 cells showed minor effects on PrCR, while the pre-treatment of BMDMs significantly induced PrCR without impacting their cell viability, suggesting that cabazitaxel played a role in directly activating macrophages (figure 3A,B; online supplemental figure 2). Macrophages pre-treated with cabazitaxel were evaluated in multiple TNBC cell models and demonstrated an enhanced phagocytic ability against all models examined, when CD47 was blocked with blocking antibodies or gene knockdown (figure 3C,D; online supplemental figure 3A-E). Interestingly, docetaxel, another taxane which promotes microtubule polymerization (figure $2 \mathrm{~B}$ ), and colchicine (online supplemental figure 3F), a taxane antagonist which induces microtubule disassembly, demonstrated no effect on PrCR, suggesting the manipulation of microtubules in macrophages is not the main cause for their enhanced phagocytic ability. This unique effect was also 


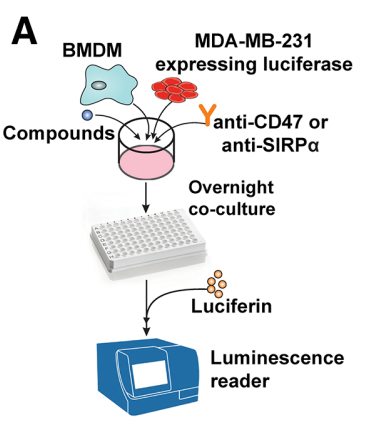

B

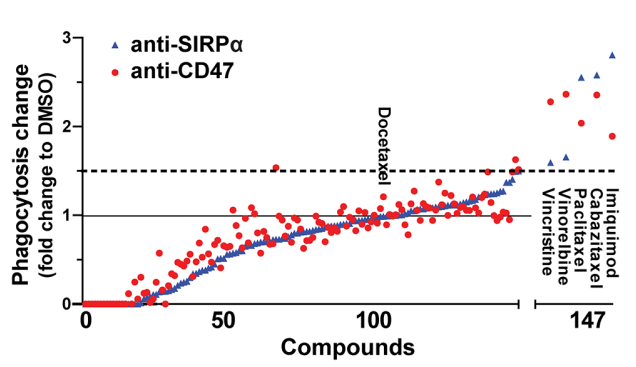

E
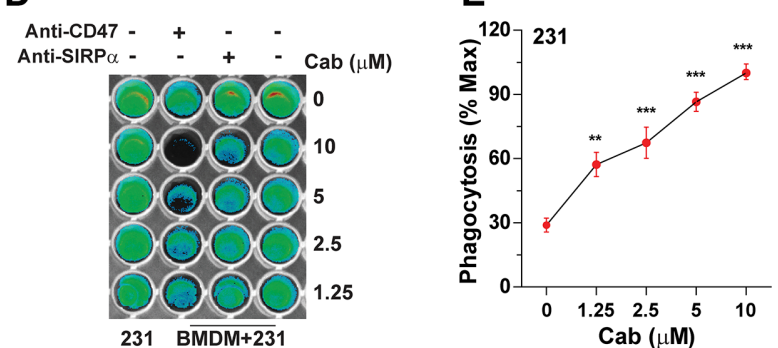
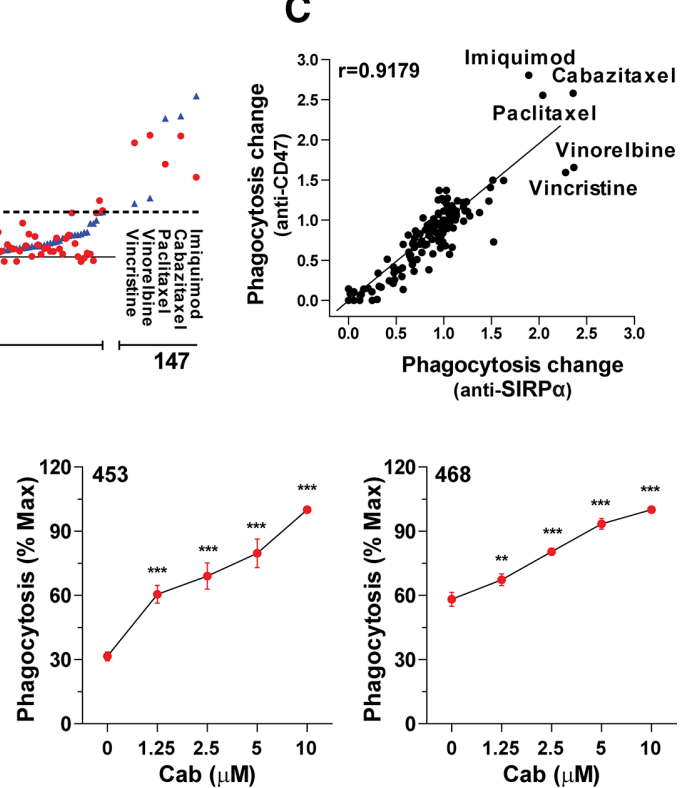

Figure 2 High-throughput screens with FDA-approved anti-cancer small molecule compounds identify cabazitaxel as a PrCR-promoting agent. (A) A schematic showing the experimental design of the high-throughput screen. (B) PrCR-based highthroughput screens of 147 FDA-approved anti-cancer small molecule compounds. Cells were treated with antibodies blocking CD47-Sirpo interaction (anti-CD47 or anti-Sirp $\alpha$ ) and subjected to luminescence-based phagocytosis assay. Phagocytosis was normalized to DMSO control. Spots represent individual compounds. (C) The correlation of phagocytosis change between screens with anti-CD47 and anti-Sirp $\alpha$ antibodies. Spots represent individual compounds. (D) Representative bioluminescence images of the luminescence-based phagocytosis assay measuring surviving cancer cells, with MDA-MB-231 cells as the target cells. Mouse M0 bone marrow-derived macrophages (BMDMs) were used for the assay. Cabazitaxel (Cab) was used at doses from 0 to $10 \mu \mathrm{M}$. (E) An in vitro luminescence-based phagocytosis assay measuring surviving cancer cells, with MDA-MB-231, MDA-MB-453 or MDA-MB-468 cells as the target cells. Mouse M0 BMDMs were used for the assay. Cells were treated with CD47-blocking antibodies and various concentrations of cabazitaxel $(0 \mu \mathrm{M}, 1.25 \mu \mathrm{M}, 2.5 \mu \mathrm{M}, 5 \mu \mathrm{M}, 10 \mu \mathrm{M})$. Phagocytosis was normalized to the maximal response in the experiments. Each group was compared with the control group $(0 \mu \mathrm{M}$ cabazitaxel). ${ }^{\star \star} \mathrm{p}<0.01,{ }^{* \star *} \mathrm{p}<0.001$ (one-way ANOVA test). Error bars represent SD.

observed in human peripheral blood monocyte-derived macrophages, in which their pre-treatment with cabazitaxel resulted in similar enhancement of CD47 blockadeinduced PrCR of TNBC cells (figure 3E), indicating the occurrence of cabazitaxel-mediated enhancement of PrCR in human macrophages.

\section{Cabazitaxel significantly enhances the efficacy of blocking CD47 in inhibiting TNBC tumor development and metastasis}

Next, we evaluated the therapeutic potential of combining cabazitaxel and CD47 blockade, which we termed "enhanced PrCR therapy", for TNBC treatment using several different preclinical mouse models. First, MDA-MB-231 cells were injected into the mammary fat pads of RAG2 ${ }^{-/-}, \gamma^{-/-}$mice which entirely lack $\mathrm{T}$, $\mathrm{B}$ and NK cells, but maintain functional macrophages. A monoclonal anti-CD47 antibody was used to block CD47-Sirp $\alpha$ interaction and induce a PrCR response. The CD47-blocking antibody and/or cabazitaxel were administered to the mice engrafted with MDA-MB-231 cells. We showed that neither CD47-blocking antibody nor cabazitaxel alone was able to significantly affect the tumor progression, whereas a combination of these two dramatically inhibited tumor development (figure 4A,B; online supplemental figure $4 \mathrm{~A}$ ), suggesting a strong synergism between these two agents. Next, syngeneic mouse TNBC models were used to evaluate the efficacy of the enhanced PrCR therapy. CD47 blockade was achieved by suppression via CRISPR/Cas9-mediated gene editing (online supplemental figure 4B). Cas9 and non-targeting sgRNA or CD47 targeting sgRNA were transduced into a mouse TNBC line, 4T1. Control knockdown $\left(\mathrm{Ctrl}^{\mathrm{KD}}\right)$ or $\mathrm{CD} 47$ knockdown $\left(\mathrm{CD} 47^{\mathrm{KD}}\right)$ 4T1 cells were injected into the mammary fat pads of immunocompetent BALB/c mice. Treatment with cabazitaxel elicited minor effects on $\mathrm{Ctrl}^{\mathrm{KD}}$ tumors but was able to significantly inhibit tumors developed by $\mathrm{CD} 47^{\mathrm{KD}} 4 \mathrm{~T} 1$ cells, which lack protection from PrCR conferred by CD47 (figure 4C,D; online supplemental figure 4C), reinforcing that cabazitaxel enhanced CD47 blockade-mediated anti-cancer activity. Depletion of TAMs by clodronate liposomes showed no effects on the burden of $\mathrm{Ctrl}^{\mathrm{KD}}$ 4T1 tumors, but largely abrogated the inhibition of tumor growth in CD47 $7^{\mathrm{KD}} 4 \mathrm{~T} 1$ tumors treated with cabazitaxel (figure 4E; online supplemental figure $4 \mathrm{D}-\mathrm{F}$ ), confirming that TAMs were the critical immune components responsible for the diminished tumor growth by the enhanced PrCR therapy. Lastly, our enhanced PrCR therapy was evaluated in a lung 

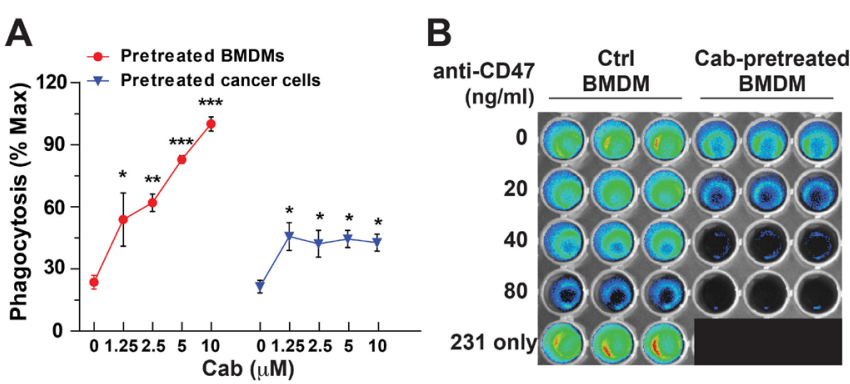

D
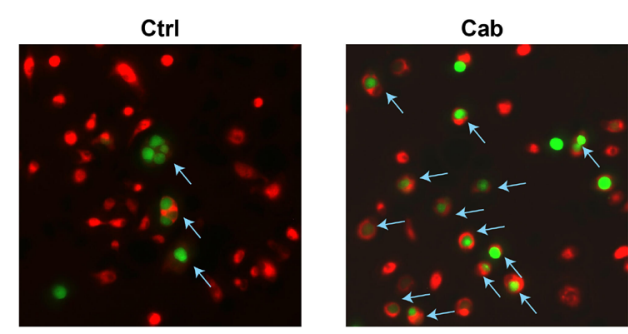

Figure 3 Cabazitaxel promotes PrCR by directly activating macrophages and independently of its cytotoxic effects toward triple-negative breast cancer (TNBC) cells. (A) An in vitro luminescence-based phagocytosis assay measuring surviving cancer cells with either TNBC cells or mouse MO bone marrow-derived macrophages (BMDMs) pre-treated with cabazitaxel. TNBC cells or BMDMs were pre-treated with various concentrations of cabazitaxel before cocultured with untreated BMDMs or TNBC cells respectively. Phagocytosis assay was performed in the presence of CD47-blocking antibodies. Phagocytosis was normalized to the maximal response in the experiments. Each group was compared with the control group $(0 \mu \mathrm{M}$ cabazitaxel). ${ }^{*} \mathrm{p}<0.05,{ }^{* *} \mathrm{p}<0.01,{ }^{* * *} \mathrm{p}<0.001$ (one-way ANOVA test). Error bars represent SD. (B) Representative bioluminescence images of the luminescence-based phagocytosis assay measuring surviving cancer cells, with MDA-MB-231 cells as the target cells. Mouse MO BMDMs pre-treated with $10 \mu \mathrm{M}$ cabazitaxel or DMSO were used for the assay. (C) An in vitro flow cytometrybased phagocytosis assay, with MDA-MB-231, MDA-MB-453 or MDA-MB-468 cells as target cells. Mouse M0 BMDMs pre-treated with DMSO or cabazitaxel were used for the assay. Phagocytosis assay was performed in the presence of CD47blocking antibodies. Phagocytosis was normalized to the maximal response in the experiments. Each group was compared with the control group $\left(0 \mu \mathrm{M}\right.$ cabazitaxel). ${ }^{*} \mathrm{p}<0.05,{ }^{* *} \mathrm{p}<0.01,{ }^{* \star *} \mathrm{p}<0.001$ (one-way ANOVA test). Error bars represent SD. (D) Representative fluorescence microscopic images of a phagocytosis assay, with CD47 ${ }^{\mathrm{KD}} \mathrm{MDA}-\mathrm{MB}-231$ cells as target cells. Mouse MO BMDMs (red) were stained with CellTrace Far Red, while CD47 ${ }^{\mathrm{KD}}$ MDA-MB-231 (green) were labeled with CellTrace Calcein Green. Arrows indicated the macrophages (double colors) that phagocytosed cancer cells. $(E)$ An in vitro luminescencebased phagocytosis assay measuring surviving cancer cells, with MDA-MB-231 cells as target cells. Human peripheral blood monocytic cell-derived macrophages were used for the assay. Phagocytosis assay was performed in the presence of CD47blocking antibodies. Phagocytosis was normalized to the maximal response in the experiments. Each group was compared with the control group $\left(0 \mu \mathrm{M}\right.$ cabazitaxel). ${ }^{\star \star} \mathrm{p}<0.01,{ }^{* \star \star} \mathrm{p}<0.001$ (one-way ANOVA test). Error bars represent SD.

metastatic model. 4T1 cells were intravenously injected to BALB/c mice, which were treated with cabazitaxel after $4 \mathrm{~T} 1$ metastases were established in the lungs. $\mathrm{CD} 47^{\mathrm{KD}} 4 \mathrm{~T} 1$ cells demonstrated a slightly compromised ability to colonize the lung and thus improved mice survival (figure $4 \mathrm{~F}-\mathrm{H}$; online supplemental figure $4 \mathrm{G}, \mathrm{H})$. Treatment with cabazitaxel had no significant effect on the tumor progression and lung colonization in mice engrafted with $4 \mathrm{~T} 1$ cells (figure $4 \mathrm{~F}-\mathrm{H}$ ); however, a combination of cabazitaxel and CD47 suppression nearly completely eradicated lung colonization of 4T1 cells on days 17 and 20 on engraftment, and therefore significantly improved the survival of the mice when compared with mice transplanted with $\mathrm{Ctrl}^{\mathrm{KD}} 4 \mathrm{~T} 1$ cells, from an average of 17.4 days to 40.9 days (figure $4 \mathrm{~F}-\mathrm{H}$ ). Taken together, our data indicate that cabazitaxel treatment enabled dramatically stronger in vivo anti-tumor activity mediated by CD47 blockades in both immunocompromised and immunocompetent settings.

\section{Cabazitaxel induces differentiation of macrophages toward M1-like state}

Next, we sought to understand the molecular mechanisms of how cabazitaxel promoted the phagocytic ability of macrophages toward TNBC cells. We showed that the cell surface expression of Sirpo or calreticulin on macrophages, known receptors for transducing "don't eat me" signals or targeting "eat me" signals on cancer cells, ${ }^{5762}$ was not affected by cabazitaxel treatment (online supplemental figure 5A, left and right). We decided to dissect the gene expression profiling in macrophages reprogrammed by cabazitaxel, using RNA sequencing. First, total RNA was extracted from vehicle-treated or cabazitaxel-treated BMDMs and subjected to RNA sequencing. A total of 452 genes were significantly upregulated and 491 genes were significantly downregulated by at least twofold (figure 5A). We then examined genes related to polarization of macrophages. TAMs are broadly classified into two groups-classically activated macrophages (M1) and alternatively activated 

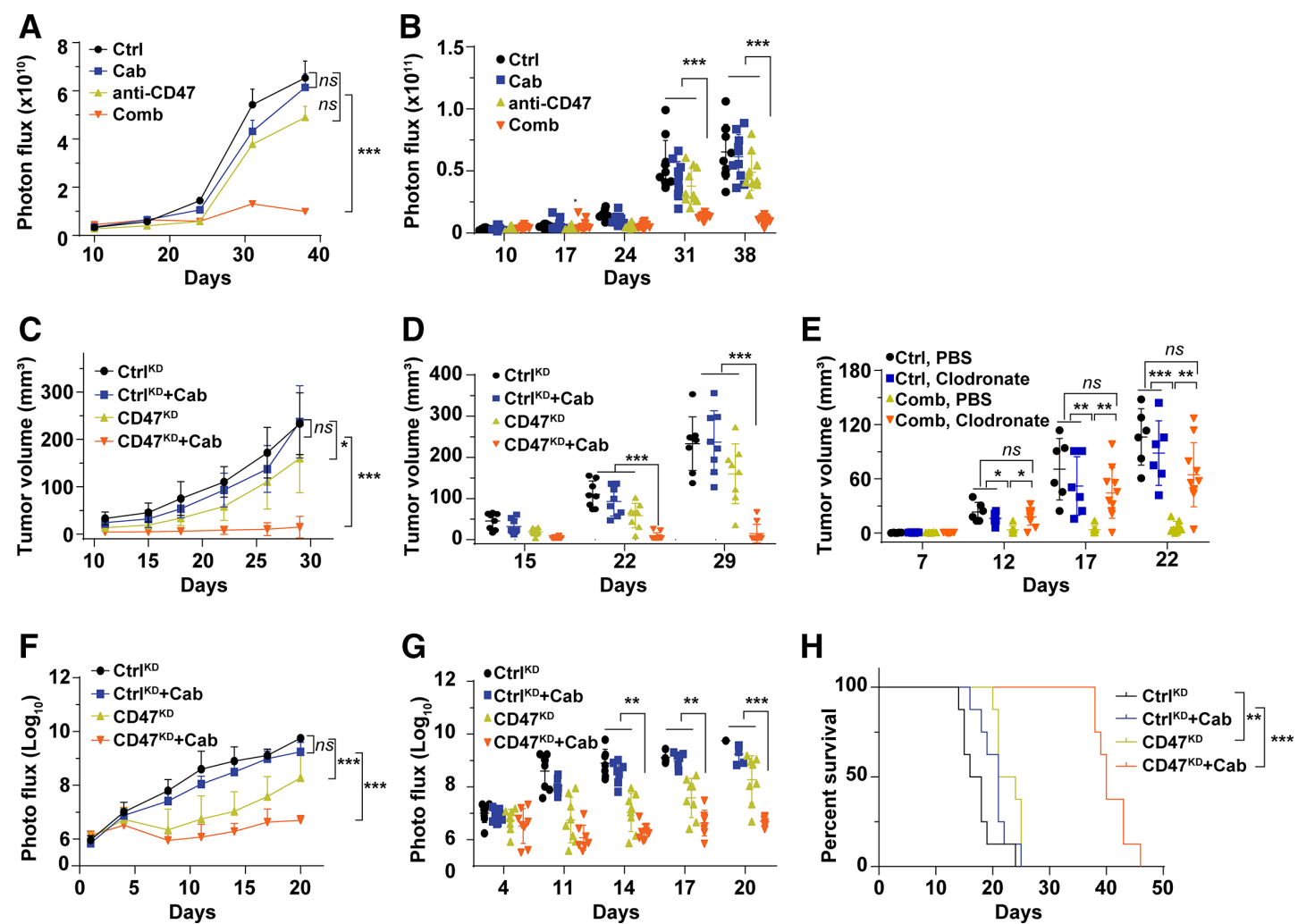

Figure 4 Cabazitaxel significantly enhances the efficacy of blocking CD47 in inhibiting triple-negative breast cancer tumor development and metastasis. (A, B) Growth of tumors developed by MDA-MB-231 cells in RAG2 $2^{-/-} \gamma \mathrm{c}^{-/-}$mice. Mice orthotopically engrafted with MDA-MB-231 cells were treated with PBS, anti-CD47 antibody, cabazitaxel or a combination of anti-CD47 antibody and cabazitaxel. Tumor growth was measured by bioluminescence imaging. (A) Tumor growth curve, ${ }^{\star \star *} \mathrm{p}<0.001$ (log-linear regression analysis). (B) Tumor burden, ${ }^{\star \star \star} \mathrm{p}<0.001$ (one-way ANOVA test). Error bars represent SD. (C, D) Growth of tumors developed by $4 \mathrm{~T} 1$ cells in BALB/c mice. Mice orthotopically engrafted with $\mathrm{Ctrl}{ }^{\mathrm{KD}}$ or CD47 ${ }^{\mathrm{KD}} 4 \mathrm{~T} 1 \mathrm{cells}$ were treated with vehicle or cabazitaxel. Tumor sizes were measured at indicated dates. (C) Tumor growth curve, ${ }^{*} 0.01<\mathrm{p}<0.05$, ${ }^{\star * *} p<0.001$ (log-linear regression analysis). (D) Tumor burden, ${ }^{*} p<0.05,{ }^{* *} p<0.01,{ }^{* * *} p<0.001$ (one-way ANOVA test). Error bars represent SD. (E) Growth of tumors developed by $4 \mathrm{~T} 1$ cells in BALB/c mice. Mice were orthotopically engrafted with $\mathrm{Ctrl}{ }^{\mathrm{KD}} 4 \mathrm{~T} 1$ and treated with vehicle (Ctrl), or were orthotopically engrafted with CD47 ${ }^{\mathrm{KD}} 4 \mathrm{~T} 1$ cells and treated with cabazitaxel (Combo). The mice were treated with control liposomes or clodronate liposomes, and tumor sizes were measured at indicated dates. ( $F$, $\mathrm{G})$ Metastasis of $4 \mathrm{~T} 1$ cells in BALB/c mice. Ctrl ${ }^{\mathrm{KD}}$ or CD47 ${ }^{\mathrm{KD}} 4 \mathrm{~T} 1$ cells were intravenously injected to the mice. Colonization and growth of 4T1 cells in the lungs were measured by bioluminescence imaging. (F) Growth curve of lung metastases, ${ }^{\star * *} \mathrm{p}<0.001$ (log-linear regression analysis). (G) Tumor burden, ${ }^{\star} \mathrm{p}<0.05,{ }^{\star \star} \mathrm{p}<0.01,{ }^{\star \star \star} \mathrm{p}<0.001$ (one-way ANOVA test). Error bars represent SD. $(\mathrm{H})$ Survival analysis of metastasis of $4 \mathrm{~T} 1$ cells in BALB/c mice, ${ }^{* *} \mathrm{p}<0.01,{ }^{\star \star *} \mathrm{p}<0.001$ (log-rank (Mantel-Cox) test).

macrophages (M2) based on their polarization states, although not always mutually exclusive. ${ }^{63}$ We compared the statistically significant changes in gene expression profiling of cabazitaxel-treated macrophages with gene signatures of M1-like and M2-like human monocytederived and mouse bone marrow-derived macrophages reported in literature. ${ }^{51} 5264$ We uncovered an upregulation of genes associated with M1-like polarization, and a downregulation of genes associated with M2-like polarization on cabazitaxel-treated BMDMs, suggesting cabazitaxel treatment elicited differentiation of BMDMs toward M1-like phenotypes (figure 5B). We then performed MDS analysis, ${ }^{65}$ using previously identified M0, M1 (polarization induced by LPS+IFN $\gamma$ ), M2 (polarization induced by IL4 or IL13) transcriptional signatures as references. ${ }^{51} 52$ Control BMDMs were clustered to M0 macrophages and on cabazitaxel treatment, the transcriptional cluster of macrophages shifted toward a M1-like signature (figure 5C). By flow cytometry analysis, we showed that on cabazitaxel-treated mouse BMDMs (figure 5D, top and bottom) and human PBMC-derived macrophages (figure $5 \mathrm{E}$, left and right), a treatment led to M1-like surface profiling with increased MHCII expression and decreased CD206 expression. In addition, when analyzing TAMs isolated from breast tumors developed by $4 \mathrm{~T} 1$ cells in BALB/c mice, we found the proportion of TAMs among myeloid or total immune cells in the tumors significantly increased in cabazitaxel-treated mice (figure 5F, left and right). Consistently, we showed an increase of MHCII and a decrease of CD206 expression on TAMs (figure 5G, left and middle; online supplemental figure $5 \mathrm{~B}$ ), as well as a significantly increased ratio of M1-like TAMs to M2-like TAMs (figure 5G, right), in the $4 \mathrm{~T} 1$ tumors from BALB/C mice receiving cabazitaxel treatment, as compared with the mice treated with vehicles. The polarization of BMDMs by IL4 to M2-like macrophages, which phenotypically resemble TAMs, decreased their 
A

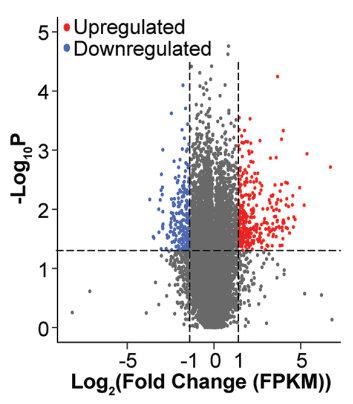

B

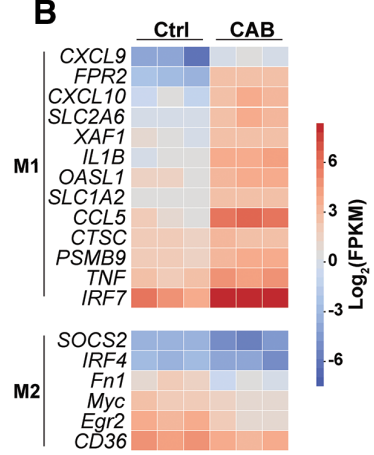

D

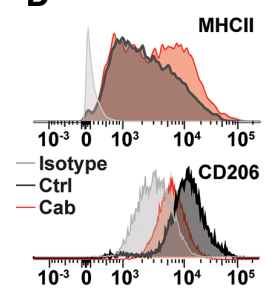

G

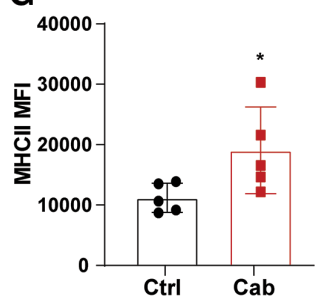

E

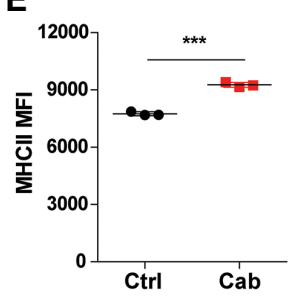

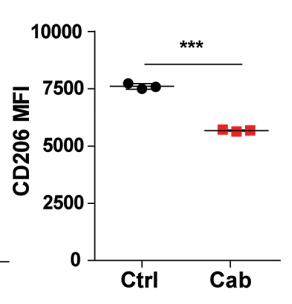

F

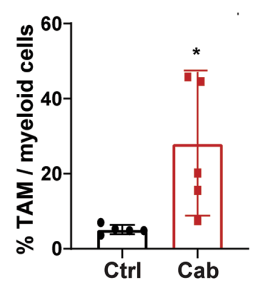

C
10

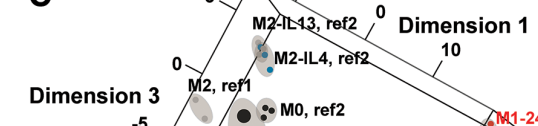

Dimension 3

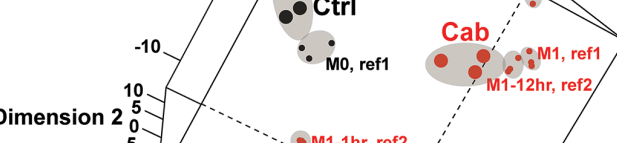

- Ctrl / MO - Cab / M1 M2, ref1 I M2-IL13, ref2 M2-IL4, ref2

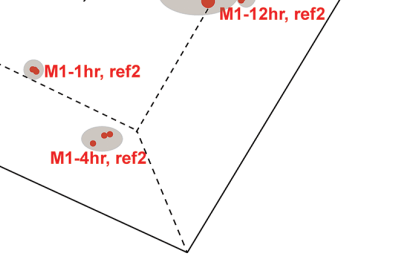

H
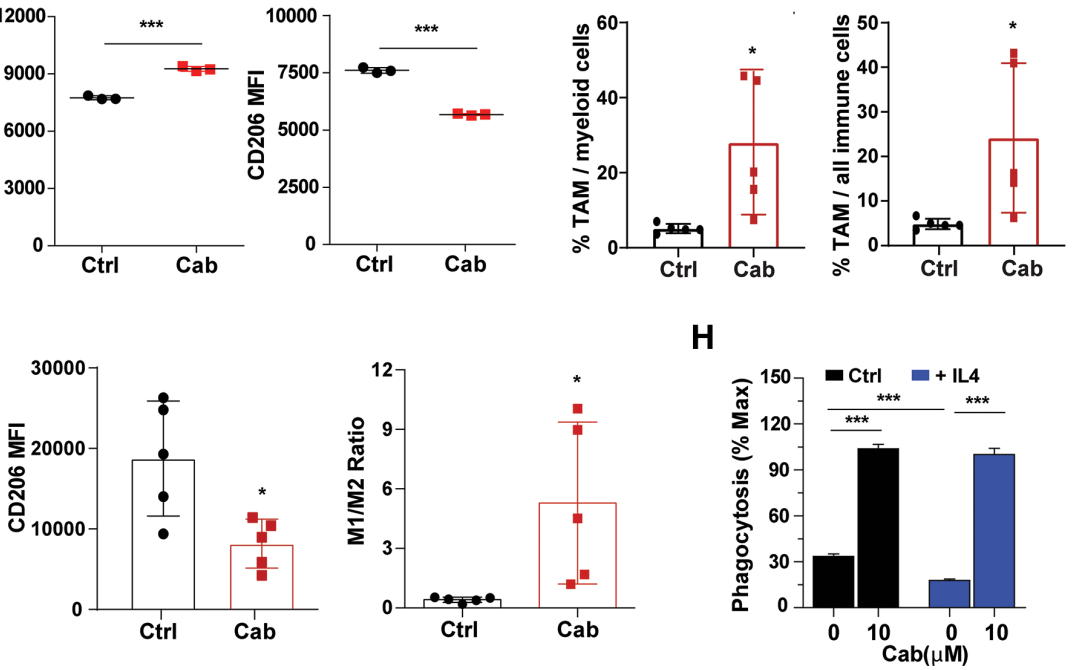

Figure 5 Cabazitaxel induces differentiation of macrophages toward M1-like state. (A) Volcano plot showing differentially expressed genes in DMSO-treated and cabazitaxel-treated bone marrow-derived macrophages (BMDMs). Genes with a p value $<0.05$ and a fold change FC $>2$ were considered as significantly upregulated or downregulated. BMDMs were generated from three individual BALB/c mice for triplicated samples. (B) A heatmap of gene expression profile by RNA sequencing showing the upregulation of M1-like gene signature and downregulation of M2-like gene signature in cabazitaxel-treated BMDMs, as compared with DMSO-treated BMDMs. (C) Multidimensional scaling (MDS) analysis of RNAseq results from DMSO-treated and cabazitaxel-treated BMDMs. Two references (ref1 and ref2) were used for MDS analysis in which M1 and M2 polarization were induced. In ref2, M1 was induced by LPS+IFNr for different time points (1, 4, 12, 24 hours) and M2 was induced by IL4 or IL13. The dots representing DMSO-treated and cabazitaxel-treated BMDMs were presented with bigger size than the dots representing the references. Replicates of the same sample are circled in a shade. (D) Representative FACS plots showing the expression of MHCII (top) and CD206 (bottom) on BMDMs stimulated with cabazitaxel or DMSO (control). (E) Mean fluorescent intensity (MFI) of MHCII (left) and CD206 (right) expression on human peripheral blood monocytic cell-derived macrophages stimulated with cabazitaxel or DMSO (control). ${ }^{* *} \mathrm{p}<0.001$ (t-test). (F) The proportion of F4/80+tumor-associated macrophages (TAMs) in CD11 $\mathrm{b}^{+}$myeloid cells, and the proportion of F4/80+CD11b+tumor-associated macrophages in $\mathrm{CD}^{+} 5^{+}$immune cells, in tumors developed by $4 \mathrm{~T} 1$ cells in BALB/c mice. Mice were treated with vehicle or cabazitaxel. ${ }^{*} \mathrm{p}<0.05$ (t-test). Error bars represent SD. (G) MFI of MHCII (left) and CD206 (middle) expression, and the ratio of M1:M2 TAMs (right) in tumors developed by $4 \mathrm{~T} 1$ cells in BALB/c mice. Mice were treated with vehicle or cabazitaxel. ${ }^{*} \mathrm{p}<0.05$ (t-test). $(\mathrm{H}) \mathrm{An}$ in vitro luminescence-based phagocytosis assay, with MDA-MB-231 cells as the target cells. Mouse M0 BMDMs were stimulated with control medium or IL4 $(10 \mathrm{ng} / \mathrm{mL}$, to induced M2 polarization) for 48 hours and then treated with DMSO or cabazitaxel. Phagocytosis assay was performed in the presence of CD47-blocking antibodies. Phagocytosis was normalized to the maximal response in the experiments. ${ }^{* \star *} \mathrm{p}<0.001$ (one-way ANOVA test). Error bars represent SD.

phagocytic ability (figure 5H). Importantly, treatment of M2-like polarized BMDMs with cabazitaxel was sufficient to restore PrCR against TNBC cells (figure 5H). Significantly, cabazitaxel-induced increased ratio of M1-like to M2-like TAMs may have profound therapeutic impact on human patients as we found with TCGA data that the increased ratio serves as an independent prognostic factor favoring patient survival in TNBC (online supplemental figure 5C).

\section{Activation of NF-kB signaling is critical for cabazitaxel- induced enhancement of PrCR}

RNA sequencing of the vehicle-treated or cabazitaxel-treated BMDMs revealed that among the pathways regulating macrophage polarization, NF-kB signaling was significantly activated in cabazitaxel-treated macrophages. Particularly, NF-kB targeted transcription factors, cytokines and chemokines were upregulated, as well as the expression of NF-kB 
A

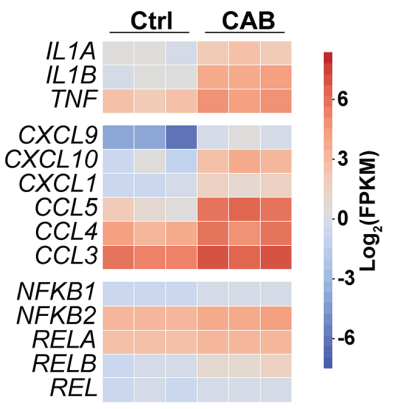

B

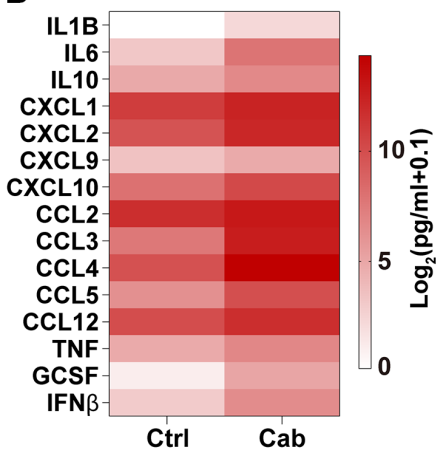

C

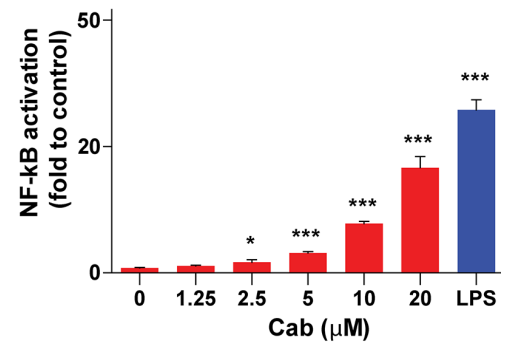

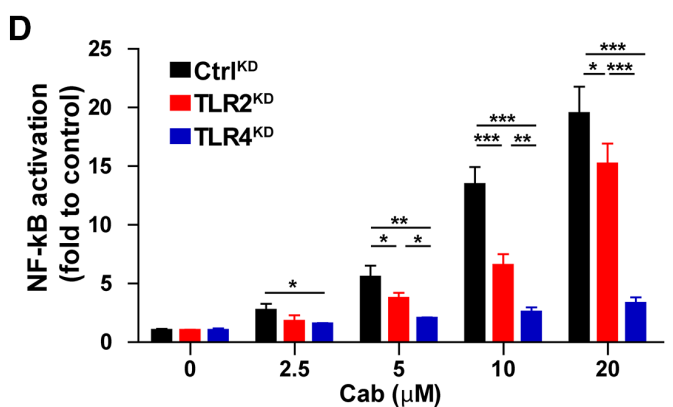

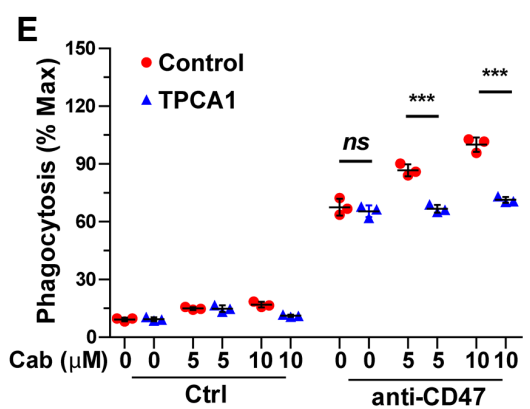

Figure 6 Activation of NF-kB signaling is critical for cabazitaxel-induced enhancement of PrCR. (A) A heatmap of gene expression prolife by RNA sequencing showing the upregulation of genes regulated or relevant to NF-kB signaling in cabazitaxel-treated bone marrow-derived macrophages (BMDMs), as compared with DMSO-treated BMDMs. (B) Measurement of cytokine and chemokine secretion profiling of BMDMs treated with DMSO (control) or cabazitaxel by a Luminex assay. Scale bars indicate $\log _{2}(\mathrm{pg} / \mathrm{mL}+0.1)$. (C) Activation of NF-kB signaling pathway, as measured by a NF-kB reporter system. RAW 264.7 macrophages were stimulated with various concentrations of cabazitaxel or $1.25 \mathrm{ng} / \mathrm{mL}$ LPS (positive control). Unstimulated RAW 264.7 cells acted as a negative control. Each group was compared with the control group $\left(0 \mu \mathrm{M}\right.$ cabazitaxel). ${ }^{*} p<0.05$, ${ }^{\star * \star} p<0.001$ (one-way ANOVA test). Error bars represent SD. (D) Activation of NF-kB signaling pathway, as measured by a NF-kB reporter system. Ctrl ${ }^{\mathrm{KD}}$, TLR2 $2^{\mathrm{KD}}$ or TLR4 ${ }^{\mathrm{KD}}$ RAW 264.7 macrophages were stimulated with various concentrations of cabazitaxel. Unstimulated RAW 264.7 cells acted as a negative control. Each group was compared with the control group $(0 \mu \mathrm{M}$ cabazitaxel). ${ }^{\star} \mathrm{p}<0.05,{ }^{* \star} \mathrm{p}<0.01,{ }^{\star \star *} \mathrm{p}<0.001$ (one-way ANOVA test). Error bars represent $\mathrm{SD}$. (E) An in vitro flow cytometry-based phagocytosis assay, with MDA-MB-231 cells as target cells. Mouse MO BMDMs were pre-treated with various concentrations of cabazitaxel in absence or presence of TPCA-1 or CD47-blocking antibodies. Phagocytosis was normalized to the maximal response in the experiments. ${ }^{* \star *} \mathrm{p}<0.001$ (one-way ANOVA test). Error bars represent SD.

family members (figure 6A). Considering the roles cytokine and chemokine molecules play in facilitating macrophage recruitment and target cell recognition and interaction, we then examined the secreted cytokine and chemokine profiling at the protein level. Consistent with our discoveries in RNAseq analysis, upon cabazitaxel treatment, a significant enhanced production of TNF, IL1B, IL6, IFN $\beta$, GCSF, CCL3, CCL4, CCL5, CXCL1, CXCL9 and CLCL10 by macrophages were observed, which are encoded by downstream targeted genes in the NF-kB pathway (figure 6B; online supplemental figure 5D). In addition, activation of NF-kB signaling was confirmed in macrophages with a NF-kB reporter system. A mouse monocyte/macrophage cell line RAW264.7 was equipped with a NF-kB reporter whose activation can drive the expression of luciferase and can be qualified by the luminescence signals. Cabazitaxel treatment drove the activation of NF-kB signaling, as indicated by the luminescence (figure 6C). Gene set enrichment analysis (GSEA) of the RNA sequencing results demonstrated the activation of innate immunity-related signaling pathways in cabazitaxel-treated macrophages, with Toll-like receptor (TLR) signaling being the most enriched pathway (online supplemental figure 6A-D). TLRs play crucial roles in activating NF-kB signaling and eliciting innate immune response. ${ }^{66}$ To further understand the molecular mechanisms by which cabazitaxel stimulated NF-kB signaling in macrophages, we used the CRISPR/Cas9 system to suppress the expression of TLRs in macrophages to examine their possible involvement. We showed that knockdown of the expression of TLR2 and TLR4 largely blocked NF-kB signaling induced by cabazitaxel, suggesting these receptors played critical roles in mediating PrCR-promoting effects of cabazitaxel in macrophages (figure 6D; online supplemental figure 6E). Treatment of macrophages with NF-kB inhibitor TPCAl which blocked IkB kinases attenuated phagocytosis and reversed cabazitaxel's effects in promoting phagocytosis, indicating that NF-kB signaling is critical for cabazitaxel-mediated enhancement of PrCR (figure 6E; online supplemental figure $6 \mathrm{~F}, \mathrm{G}$ ).

\section{DISCUSSION}

During cancer progression, conveying inhibitory signals to the immune system improves the chances of malignant cells 
in surviving immunosurveillance, leading to the dominance of cancer cells that possess or have acquired immune evasive mechanisms in the tumors. ${ }^{67}$ The identification of immune checkpoints and invention of agents targeting signals that inhibit an immune response have revolutionized the treatment of multiple types of human cancers, including those that are very aggressive and advanced. ${ }^{1068}$

A combination of atezolizumab and nab-paclitaxel led to significantly improved overall survival in patients with PD-L1+TNBC, demonstrating the efficacy of immunotherapy in treating this subgroup of aggressive breast cancers. ${ }^{3}{ }^{13}$ However, the recent Impassion 131 study (NCT03125902) revealed that paclitaxel in combination with atezolizumab failed to prolong progression-free survival of patients with TNBC when comparing with paclitaxel plus placebo, ${ }^{69}$ underscoring the need to better understand the cancer and immune system interactions. Nevertheless, the only FDA-approved immunotherapy for TNBC, atezolizumab, holds potential for improving patient prognosis or even a cure for a group of patients with TNBC where other therapies have been rendered ineffective and unsuccessful. Critical factors that may contribute to determining the overall efficacy of atezolizumab for TNBC include the expression levels of PD-L1 in tumors, lymphocytic tumor infiltration and tumor mutational burden. ${ }^{1316} 17$ Although some PD-L1 cancers also showed a response to atezolizumab, this may be partially attributed to the highly heterogeneous nature of TNBC tumors.

The fact that a large group of patients with TNBC resistant to chemotherapy showed little to no response to atezolizumab-chemotherapy combination treatment ${ }^{3}$ highlighted an urgent need for the development of additional effective treatment approaches. Given that TNBC tumors have a more ubiquitous upregulation of $\mathrm{CD} 47$ and are highly infiltrated by macrophages, this makes PrCR-based therapy a promising new class of immunotherapy for TNBC. More importantly, PrCR relies on TAMs that are abundant in the tumor microenvironment, and its execution is via mechanisms distinct from PDL1-PD1 signaling pathways; therefore, an induction of PrCR may yield effective therapies that complement or synergize with T-cell immune checkpoint inhibitors.

Recent progress from clinical trials demonstrated significant clinical benefits of using a CD47-blocking antibody on patients with non-Hodgkin's lymphoma with no clinically significant safety risks, demonstrating the potential for the restoration of macrophage-mediated PrCR as an effective cancer immunotherapy. ${ }^{32}$ Efficient activation of PrCR is critical for therapies exploiting the tumoricidal role of macrophages. ${ }^{22} 253334$ Targeting "don't eat me" signals on cancer cells remains the most effective approach for inducing PrCR, and CD47 is thus far the only "don't eat me" signal whose therapeutic potential has been evaluated in a clinical setting for multiple cancers. ${ }^{25}{ }^{31-34}$ Blockade of the negative signaling transduced by the CD47-SIRP $\alpha$ axis re-establishes macrophage-mediated immunosurveillance. ${ }^{23-25} 7071$ Few studies, however, have addressed therapeutic strategies for the treatment of TNBC by targeting TAMs, which are especially highly enriched in TNBC tumors.
Here, we show that the blockade of CD47 induced PrCR of TNBC cells, but its efficacy needs to be improved to meet the demands for an effective therapy. In addition to this, previous studies indicated that blocking antibodies need to reach a local concentration threshold in order to effectively activate $\operatorname{PrCR},{ }^{72}$ which is often difficult to achieve, especially in solid tumors. Therefore, agents that can penetrate tumor tissues to directly activate TAMs are attractive candidates for improving the efficacy and feasibility of PrCR induction. Using anti-cancer small molecule compounds with a proven tumor-penetrating ability becomes an attractive strategy for promoting PrCR directly. Here, we demonstrate that the chemotherapeutic agent cabazitaxel can be reprofiled as an immune adjuvant to directly enhance PrCR ability of macrophages. Cabazitaxel was approved by FDA as a chemotherapy for treating prostate cancers and exerts its cytotoxicity by stabilizing tubulin in the microtubule to inhibit cell division. ${ }^{39}$ Surprisingly, our results demonstrated that the anti-cancer effects of cabazitaxel in TNBC resulted from the activation of macrophages rather than direct cytotoxicity toward TNBC cells. Cabazitaxel induces polarization of macrophages toward a M1 state, as demonstrated by the activation of TLR/NF-kB pathways and enhanced expression of pro-inflammatory cytokines. Interestingly, paclitaxel has been shown to be a TLR4 ligand which directly binds to myeloid differentiation factor 2 (MD-2), a TLR4 accessory protein, and activates downstream signaling. ${ }^{73}$ Further investigation may be performed to dissect the molecular mechanisms by which cabazitaxel interacts with and activates TLRs. Importantly, M2-polarized macrophages can be converted to be M1-like by using cabazitaxel to acquire a significantly improved phagocytic ability, revealing a plastic and inducible nature of intrinsic phagocytic machinery and its regulator programs. A recent phase II clinical trial demonstrated that cabazitaxel is active (disease control rate of almost of $60 \%$ ) with a manageable toxicity profile with HER2-negative metastatic breast cancer, ${ }^{74}$ further suggesting the feasibility of combining cabazitaxel with CD47 blockades in TNBC treatment.

Taken together, our study revealed the significant therapeutic potential of combining cabazitaxel and CD47 blockade, and uncovered a promising strategy for targeting TAMs for the treatment of TNBC, including metastatic TNBC. Next, further analysis at the single-cell level may be performed to characterize TAM subpopulations and identify populations that are sensitive to cabazitaxel, with the most potential for phagocytosis on administration of treatment, which may serve as a predictive parameter for selecting patients with TNBC most likely to benefit from the enhanced PrCR therapy.

\section{Author affiliations}

${ }^{1}$ Department of Immuno-Oncology, Beckman Research Institute, City of Hope, Duarte, California, USA

${ }^{2}$ Department of Hematology and Hematopoietic Cell Transplantation, City of Hope, Duarte, California, USA

${ }^{3}$ Department of Surgery, City of Hope, Duarte, California, USA

${ }^{4}$ Division of Dermatology, City of Hope, Duarte, California, USA

${ }^{5}$ Department of Pathology, City of Hope, Duarte, California, USA 
${ }^{6}$ Hematologic Malignancies and Stem Cell Transplantation Institute, City of Hope, Duarte, California, USA

${ }^{7}$ Beckman Research Institute, City of Hope, Duarte, California, USA

${ }^{8}$ Center for Informatics, City of Hope, Duarte, California, USA

Contributors XC: Project conceptualization, validation, experimental design, investigation, methodology, writing-original draft, writing-review and editing. BL: Project conceptualization, validation, experimental design, investigation, methodology. JC: Project conceptualization, validation, experimental design, investigation, methodology. JD: Experimental design, investigation, methodology. SC: Experimental design, investigation, methodology. EGG: Experimental design, scientific input. BX: Experimental design, scientific input. LT: Experimental design, scientific input. SM: Experimental design, scientific input. MR: Experimental design, scientific input. CQ: Scientific input, supervision. JY: Scientific input, supervision. STR: Scientific input, supervision. YW: Project conceptualization, validation, investigation, methodology, bioinformatics and RNAseq data analysis, writingoriginal draft, writing-review and editing. MF: Project conceptualization, supervision, funding acquisition, experimental design, investigation, project administration, writing-original draft, writing-review and editing.

Funding We thank the excellent technical support of Core Facilities at City of Hope, including the Analytical Cytometry, Light Microscopy, Animal Resource Center and Small Animal Imaging Core Facilities supported by the National Cancer Institute of the National Institutes of Health under award number P30CA033572. This work was supported by the National Cancer Institute of the National Institutes of Health K99/ R00 Pathway to Independence Award K99CA201075/R00CA201075 (to MF) and CA247550 (to JY), the National Institute of Allergy and Infectious Diseases of the National Institutes of Health Al129582 (to JY), the Leukemia \& Lymphoma Society 1364-19 (to JY), the Damon Runyon-Dale F. Frey Award for Breakthrough Scientists DFS-22-16 (to MF), the V Foundation for Cancer Research V Scholar Award (V2018012) (MF), and the startup research funding from City of Hope (to MF). Funds from an anonymous donor helped accelerate these studies.

Competing interests MF declares patent applications pertaining to stimulating TLR/BTK signaling to promote CRT in macrophages assigned to the Stanford University and equity and/or consulting with Forty Seven, Inc.

Patient consent for publication Not required.

Provenance and peer review Not commissioned; externally peer reviewed.

Data availability statement Data are available on reasonable request.

Supplemental material This content has been supplied by the author(s). It has not been vetted by BMJ Publishing Group Limited (BMJ) and may not have been peer-reviewed. Any opinions or recommendations discussed are solely those of the author(s) and are not endorsed by BMJ. BMJ disclaims all liability and responsibility arising from any reliance placed on the content. Where the content includes any translated material, BMJ does not warrant the accuracy and reliability of the translations (including but not limited to local regulations, clinical guidelines, terminology, drug names and drug dosages), and is not responsible for any error and/or omissions arising from translation and adaptation or otherwise.

Open access This is an open access article distributed in accordance with the Creative Commons Attribution Non Commercial (CC BY-NC 4.0) license, which permits others to distribute, remix, adapt, build upon this work non-commercially, and license their derivative works on different terms, provided the original work is properly cited, appropriate credit is given, any changes made indicated, and the use is non-commercial. See http://creativecommons.org/licenses/by-nc/4.0/.

\section{ORCID iDs}

E Gulsen Gunes http://orcid.org/0000-0002-4817-9316

Mingye Feng http://orcid.org/0000-0002-3334-9917

\section{REFERENCES}

1 Bianchini G, Balko JM, Mayer IA, et al. Triple-negative breast cancer: challenges and opportunities of a heterogeneous disease. Nat Rev Clin Oncol 2016;13:674-90.

2 Garrido-Castro AC, Lin NU, Polyak K. Insights into molecular classifications of triple-negative breast cancer: improving patient selection for treatment. Cancer Discov 2019;9:176-98.

3 Schmid P, Adams S, Rugo HS, et al. Atezolizumab and nabpaclitaxel in advanced triple-negative breast cancer. N Engl J Med 2018;379:2108-21.
4 Coulie PG, Van den Eynde BJ, van der Bruggen P, et al. Tumour antigens recognized by $T$ lymphocytes: at the core of cancer immunotherapy. Nat Rev Cancer 2014;14:135-46.

5 Ruella M, Kalos M. Adoptive immunotherapy for cancer. Immunol Rev 2014;257:14-38.

6 Cheadle EJ, Gornall H, Baldan V, et al. Car T cells: driving the road from the laboratory to the clinic. Immunol Rev 2014;257:91-106.

7 Vyas M, Koehl U, Hallek M, et al. Natural ligands and antibody-based fusion proteins: harnessing the immune system against cancer. Trends Mol Med 2014;20:72-82.

8 Jackson HJ, Rafiq S, Brentjens RJ. Driving CAR T-cells forward. Nat Rev Clin Oncol 2016;13:370-83.

9 Wei SC, Duffy CR, Allison JP. Fundamental mechanisms of immune checkpoint blockade therapy. Cancer Discov 2018;8:1069-86.

10 Topalian SL, Drake CG, Pardoll DM. Immune checkpoint blockade: a common denominator approach to cancer therapy. Cancer Cell 2015;27:450-61.

11 Mittendorf EA, Philips AV, Meric-Bernstam F, et al. Pd-L1 expression in triple-negative breast cancer. Cancer Immunol Res 2014;2:361-70.

12 Loi S, Michiels S, Salgado R, et al. Tumor infiltrating lymphocytes are prognostic in triple negative breast cancer and predictive for trastuzumab benefit in early breast cancer: results from the FinHER trial. Ann Oncol 2014;25:1544-50.

13 Marra A, Viale G, Curigliano G. Recent advances in triple negative breast cancer: the immunotherapy era. BMC Med 2019;17:90.

14 Narayan P, Wahby S, Gao JJ, et al. FDA approval summary: atezolizumab plus paclitaxel protein-bound for the treatment of patients with advanced or metastatic TNBC whose tumors express PD-L1. Clin Cancer Res 2020;26:2284-9.

15 Cyprian FS, Akhtar S, Gatalica Z, et al. Targeted immunotherapy with a checkpoint inhibitor in combination with chemotherapy: a new clinical paradigm in the treatment of triple-negative breast cancer. Bosn J Basic Med Sci 2019;19:227-33.

16 Emens LA, Cruz C, Eder JP, et al. Long-term clinical outcomes and biomarker analyses of atezolizumab therapy for patients with metastatic triple-negative breast cancer: a phase 1 study. JAMA Oncol 2019;5:74-82.

17 Kwa MJ, Adams S. Checkpoint inhibitors in triple-negative breast cancer (TNBC): where to go from here. Cancer 2018;124:2086-103.

18 Franklin RA, Liao W, Sarkar A, et al. The cellular and molecular origin of tumor-associated macrophages. Science 2014;344:921-5.

19 Noy R, Pollard JW. Tumor-associated macrophages: from mechanisms to therapy. Immunity 2014;41:49-61.

20 Ruffell B, Coussens LM. Macrophages and therapeutic resistance in cancer. Cancer Cell 2015;27:462-72.

21 Qian B-Z, Li J, Zhang H, et al. Ccl2 recruits inflammatory monocytes to facilitate breast-tumour metastasis. Nature 2011;475:222-5.

22 Cassetta L, Pollard JW. Targeting macrophages: therapeutic approaches in cancer. Nat Rev Drug Discov 2018;17:887-904.

23 Jaiswal S, Chao MP, Majeti R, et al. Macrophages as mediators of tumor immunosurveillance. Trends Immunol 2010;31:212-9.

24 Chao MP, Majeti R, Weissman IL. Programmed cell removal: a new obstacle in the road to developing cancer. Nat Rev Cancer 2011;12:58-67.

25 Feng M, Jiang W, Kim BYS, et al. Phagocytosis checkpoints as new targets for cancer immunotherapy. Nat Rev Cancer 2019;19:568-86.

26 Majeti R, Chao MP, Alizadeh AA, et al. CD47 is an adverse prognostic factor and therapeutic antibody target on human acute myeloid leukemia stem cells. Cell 2009;138:286-99.

27 Willingham SB, Volkmer J-P, Gentles AJ, et al. The CD47-signal regulatory protein alpha (SIRPa) interaction is a therapeutic target for human solid tumors. Proc Natl Acad Sci U S A 2012;109:6662-7.

28 Barkal AA, Weiskopf K, Kao KS, et al. Engagement of MHC class I by the inhibitory receptor LILRB1 suppresses macrophages and is a target of cancer immunotherapy. Nat Immunol 2018;19:76-84.

29 Barkal AA, Brewer RE, Markovic M, et al. Cd24 signalling through macrophage Siglec-10 is a target for cancer immunotherapy. Nature 2019;572:392-6.

30 Jaiswal S, Jamieson CHM, Pang WW, et al. Cd47 is upregulated on circulating hematopoietic stem cells and leukemia cells to avoid phagocytosis. Cell 2009;138:271-85.

31 Sikic BI, Lakhani N, Patnaik A, et al. First-in-human, first-in-class phase I trial of the anti-CD47 antibody Hu5F9-G4 in patients with advanced cancers. J Clin Oncol 2019;37:946-53.

32 Advani R, Flinn I, Popplewell L, et al. Cd47 blockade by Hu5F9-G4 and rituximab in non-Hodgkin's lymphoma. $N$ Engl J Med 2018;379:1711-21.

33 Jalil AR, Andrechak JC, Discher DE. Macrophage checkpoint blockade: results from initial clinical trials, binding analyses, and CD47-SIRP $\alpha$ structure-function. Antib Ther 2020;3:80-94. 
34 Russ A, Hua AB, Montfort WR, et al. Blocking "don't eat me" signal of CD47-SIRP $\alpha$ in hematological malignancies, an in-depth review. Blood Rev 2018;32:480-9.

35 Chan KS, Espinosa I, Chao M, et al. Identification, molecular characterization, clinical prognosis, and therapeutic targeting of human bladder tumor-initiating cells. Proc Natl Acad Sci U S A 2009:106:14016-21.

36 Petrova PS, Viller NN, Wong M, et al. TTI-621 (SIRP $\alpha \mathrm{Fc}$ ): a CD47-blocking innate immune checkpoint inhibitor with broad antitumor activity and minimal erythrocyte binding. Clin Cancer Res 2017;23:1068-79.

37 Xiao Z, Chung H, Banan B, et al. Antibody mediated therapy targeting CD47 inhibits tumor progression of hepatocellular carcinoma. Cancer Lett 2015;360:302-9.

38 Yuan J, Shi X, Chen C, et al. High expression of CD47 in triple negative breast cancer is associated with epithelial-mesenchymal transition and poor prognosis. Oncol Lett 2019;18:3249-55.

39 Tsao C-K, Cutting E, Martin J, et al. The role of cabazitaxel in the treatment of metastatic castration-resistant prostate cancer. Ther Adv Urol 2014;6:97-104.

40 Goldman MJ, Craft B, Hastie M, et al. Visualizing and interpreting cancer genomics data via the Xena platform. Nat Biotechnol 2020;38:675-8.

41 Hatzis C, Pusztai L, Valero V, et al. A genomic predictor of response and survival following taxane-anthracycline chemotherapy for invasive breast cancer. JAMA 2011;305:1873-81.

42 Jézéquel P, Loussouarn D, Guérin-Charbonnel C, et al. Geneexpression molecular subtyping of triple-negative breast cancer tumours: importance of immune response. Breast Cancer Res 2015;17:43

43 Burstein MD, Tsimelzon A, Poage GM, et al. Comprehensive genomic analysis identifies novel subtypes and targets of triple-negative breast cancer. Clin Cancer Res 2015;21:1688-98.

44 Sabatier R, Finetti P, Cervera N, et al. A gene expression signature identifies two prognostic subgroups of basal breast cancer. Breast Cancer Res Treat 2011;126:407-20.

45 Newman AM, Liu CL, Green MR, et al. Robust enumeration of cell subsets from tissue expression profiles. Nat Methods 2015;12:453-7.

46 Sanjana NE, Shalem O, Zhang F. Improved vectors and genomewide libraries for CRISPR screening. Nat Methods 2014;11:783-4.

47 Qi LS, Larson MH, Gilbert LA, et al. Repurposing CRISPR as an RNA-guided platform for sequence-specific control of gene expression. Cell 2013;152:1173-83.

48 Covarrubias S, Robinson EK, Shapleigh B, et al. CRISPR/Cas-based screening of long non-coding RNAs (IncRNAs) in macrophages with an NF- $\kappa$ B reporter. J Biol Chem 2017;292:20911-20.

49 Mootha VK, Lindgren CM, Eriksson K-F, et al. PGC-1alpharesponsive genes involved in oxidative phosphorylation are coordinately downregulated in human diabetes. Nat Genet 2003:34:267-73.

50 Subramanian A, Tamayo P, Mootha VK, et al. Gene set enrichment analysis: a knowledge-based approach for interpreting genome-wide expression profiles. Proc Natl Acad Sci U S A 2005;102:15545-50.

51 Das A, Yang C-S, Arifuzzaman S, et al. High-resolution mapping and dynamics of the transcriptome, transcription factors, and transcription co-factor networks in classically and alternatively activated macrophages. Front Immunol 2018;9:22.

52 Jablonski KA, Amici SA, Webb LM, et al. Novel markers to delineate murine M1 and M2 macrophages. PLoS One 2015;10:e0145342.

53 Ho CCM, Guo N, Sockolosky JT, et al. "Velcro" engineering of high affinity CD47 ectodomain as signal regulatory protein $\alpha(\mathrm{SIRP} \alpha)$ antagonists that enhance antibody-dependent cellular phagocytosis. J Biol Chem 2015;290:12650-63.
54 Iwamoto C, Takenaka K, Urata S, et al. The BALB/c-specific polymorphic SIRPa enhances its affinity for human CD47, inhibiting phagocytosis against human cells to promote xenogeneic engraftment. Exp Hematol 2014;42:163-71.

55 Iwamoto $\mathrm{C}$, Takenaka K, Urata S, et al. The efficient engraftment of human hematopoiesis in the BALB/c strain is mounted by Balb/cSpecific SIRPa polymorphism that enhances binding affinity to human CD47. Blood 2011;118:4008

56 Chao MP, Alizadeh AA, Tang C, et al. Anti-CD47 antibody synergizes with rituximab to promote phagocytosis and eradicate non-Hodgkin lymphoma. Cell 2010;142:699-713.

57 Feng M, Chen JY, Weissman-Tsukamoto R, et al. Macrophages eat cancer cells using their own calreticulin as a guide: roles of TLR and Btk. Proc Natl Acad Sci U S A 2015;112:2145-50.

58 Obeid M, Tesniere A, Ghiringhelli F, et al. Calreticulin exposure dictates the immunogenicity of cancer cell death. Nat Med 2007;13:54-61.

59 Abu Eid R, Razavi GSE, Mkrtichyan M, et al. Old-school chemotherapy in immunotherapeutic combination in cancer, a lowcost drug repurposed. Cancer Immunol Res 2016;4:377-82.

60 de Morrée E, van Soest R, Aghai A, et al. Understanding taxanes in prostate cancer; importance of intratumoral drug accumulation. Prostate 2016;76:927-36.

61 de Wit R, de Bono J, Sternberg CN, et al. Cabazitaxel versus abiraterone or enzalutamide in metastatic prostate cancer. $N$ Engl $J$ Med 2019;381:2506-18.

62 Feng M, Marjon KD, Zhu F, et al. Programmed cell removal by calreticulin in tissue homeostasis and cancer. Nat Commun 2018;9:3194.

63 Locati M, Curtale G, Mantovani A. Diversity, mechanisms, and significance of macrophage plasticity. Annu Rev Pathol 2020;15:123-47.

64 Martinez FO, Gordon S, Locati M, et al. Transcriptional profiling of the human monocyte-to-macrophage differentiation and polarization: new molecules and patterns of gene expression. J Immunol 2006;177:7303-11.

65 Franks JM, Cai G, Whitfield ML. Feature specific quantile normalization enables cross-platform classification of molecular subtypes using gene expression data. Bioinformatics 2018;34:1868-74.

66 O'Neill LAJ, Golenbock D, Bowie AG. The history of Tolllike receptors - redefining innate immunity. Nat Rev Immunol 2013;13:453-60

67 Hanahan D, Weinberg RA. Hallmarks of cancer: the next generation. Cell 2011:144:646-74.

68 Sharma P, Allison JP. The future of immune checkpoint therapy. Science 2015;348:56-61.

69 Esteva FJ, Hubbard-Lucey VM, Tang J, et al. Immunotherapy and targeted therapy combinations in metastatic breast cancer. Lancet Oncol 2019;20:e175-86.

70 Wang $\mathrm{H}$, VerHalen J, Madariaga ML, et al. Attenuation of phagocytosis of xenogeneic cells by manipulating CD47. Blood 2007; 109:836-42.

71 Oldenborg PA, Zheleznyak A, Fang YF, et al. Role of CD47 as a marker of self on red blood cells. Science 2000;288:2051-4.

72 Ingram JR, Blomberg OS, Sockolosky JT, et al. Localized CD47 blockade enhances immunotherapy for murine melanoma. Proc Nat Acad Sci U S A 2017;114:10184-9.

73 Zimmer SM, Liu J, Clayton JL, et al. Paclitaxel binding to human and murine MD-2. J Biol Chem 2008;283:27916-26.

74 Koutras A, Zagouri F, Koliou G-A, et al. Phase II study of cabazitaxel as second-line treatment in patients with HER-2 negative metastatic breast cancer previously treated with taxanes. JCO 2018;36:1089 